NASA Contractor Report 4264

\title{
The Radiation of Sound From \\ a Propeller at Angle of Attack
}

Ramani Mani

CONTRACT NAS3-23721

JANUARY 1990 
NASA Contractor Report 4264

\title{
The Radiation of Sound From \\ a Propeller at Angle of Attack
}

\author{
Ramani Mani \\ General Electric Company \\ Scbenectady, New York
}

Prepared for

Lewis Research Center

under Contract NAS3-23721

\section{N/Sก}

National Aeronautics and

Space Administration

Office of Management

Scientific and Technical

Intormation Division

1990 



\section{$\underline{\text { Summary }}$}

The mechanism by which the noise generated at the blade passing frequency by a propeller is altered when the propeller axis is at an angle of attack to the freestream is examined herein. The measured noise field is distinctly non axially symmetric under such conditions with far field sound pressure levels both diminished and increased relative to the axially symmetric values produced with the propeller at zero angle of attack. Attempts have been made to explain this non axially symmetric sound field based on the unsteady ("once per rev") loading experienced by the propeller blades when the propeller axis is at non zero angle of attack. A calculation based on this notion appears to greatly underestimate the measured azimuthal asymmetry of noise for high tip speed, highly loaded propellers. A new mechanism is proposed herein; namely, that at angle of attack, there is a non axially symmetric modulation of the radiative efficiency of the steady loading and thickness noise which is the primary cause of the non axially symmetric sound field at angle of attack for high tip speed, heavily loaded propellers with a large number of blades. A calculation of this effect to first order in the crossflow Mach number (component of freestream Mach number normal to the propeller axis) is carried out and shows much better agreement with measured noise data on the angle of attack effect. 
$\underline{\text { Introduction }}$

In recent years, there has been a renewed interest in high tip speed turboprop systems for commercial aircraft applications. Many of the practical applications envisage counter rotating propeller (CRP) driven aircraft though careful experimental evaluations (both aerodynamic and acoustic) have been made of both single rotation propellers (SRP) and CRP's.

In the present study, we address a particular aspect of the noise radiated at the blade passing frequency (BPF) by a propeller. In an ideally uniform stream with the propeller axis coincident with the direction of the free stream, aerodynamic sound at the BPF produced by the propeller blades is due to the steady air loading (drag and lift) carried by the blades and also due to the thickness of the blades. A characteristic of this sound field is that it is axially symmetric in magnitude with the axis of symmetry being the propeller axis. The first theoretical analysis of steady loading noise was given by Gutin (1936) with Garrick \& Watkins (1954) contributing the extension of Gutin's work to the case of a propeller in forward flight. Much progress has been made since these studies in terms of our ability to predict steady loading/thickness related noise. The two principal avenues of improvement in recent years have been the use of computational fluid mechanics techniques to predict the steady pressure distribution on the blade surfaces and the recognition in the acoustic theory of source noncompactness effects.

When the propeller is installed in an aircraft, clearly it does not operate in the environment of a uniform stream whose direction is coincident with the propeller axis and the sound field at the BPF can be quite different from the previously described ideal case. These effects are described as "installation effects". An elementary example of the installation effect is that under takeoff/approach conditions, the direction of the propeller axis deviates from the direction of forward flight leading to the "angle of attack" effect. Recent experimental studies of the angle of attack effect on propeller BPF noise include those of Block (1984) and Woodward (1987a) for SRP's and of Woodward (1987b) for CRP's. Paradoxically, as will be discussed below, this most elementary installation effect has proved 
quite difficult to understand.

The characteristics of the measured non axially symmetric sound field at angle of attack can be described in terms of the polar coordinate system shown in figure 1. The BPF sound field (which is axially symmetric at zero angle of attack) roughly speaking is found to be diminished in the upper quadrants on the right hand half of Figure 1 (i.e. for $0 \leq \phi \leq \pi$ ) and increased in the lower quadrant ( $\pi$ $\leq \phi \leq 2 \pi$ ) with the minima and maxima occurring approximately at $\phi=\pi / 2$ and $\phi=3 \pi / 2$ relative to the zero angle of attack case. For highly loaded, high tip speed propellers the changes can be quite large. In the vicinity of the plane of rotation the changes can be as large as $1 \mathrm{~dB}$ per degree of angle of attack. All prior attempts to explain this noise change due to angle of attack have proceeded as follows. The BPF noise of the propeller due to steady loading and thickness is calculated using the usual methods for a propeller in uniform axial flow. This sound field due to steady loading/thickness which is axisymmetric in amplitude but not in phase is assumed to be unchanged with the propeller operating at angle of attack. Due to angle of attack, clearly unsteady loading at "one per revolution" will be experienced by the propeller blades which will cause additional noise at the BPF. When this new sound field is added to that due to the steady loading with proper consideration being given to the phasing between the two sources, a resultant non axisymmetric sound field is obtained.

As will be shown in the next section, for lightly loaded propellers especially with few blades and spinning at low tip speeds, this procedure does seem to yield results in agreement with data. For highly loaded, high tip speed propellers especially with larger number of blades, this procedure yields gross underestimates of the measured departure from the axisymmetrical situation due to angle of attack. A new mechanism is pointed out which negates the notion that the sound field due to steady loading/thickness is unchanged by angle of attack. A first order (in angle of attack) estimation of this mechanism is carried out which appears to greatly improve the theory-data comparisons. Indeed the suggestion that emerges from this analysis is that for highly loaded, high tip speed propellers with a large number of blades, the angle of attack effect on BPF propeller noise is largely unrelated to 
unsteady loading.

The notation used in this report is indicated in Appendix 2. 
Steady Loading and Unsteady Loading Noise

In the work described herein, the acoustic source distributions are assumed to be acoustically compact in the chordwise direction. In other words, a line source model is employed. Also in this section the only mean flow effect accounted for is a uniform axial flow corresponding to the flight velocity. At time " $t=0$, let the location of this line source be as shown in Figure 4, and let the sweep be as shown in Figure 5. Thus, " $\alpha_{1}(r)$ " and " $\Delta x(r)$ " define the radial lean and axial sweep of the blades. The following results were derived for the purposes of this work by the use of axial Fourier transforms and the method of stationary phase, but they are in agreement with results given by Hawkings and Lowson (1974) and Hanson (1984), and hence, only the final result of the analysis is given.

The contribution to $\mathrm{p}_{\mathrm{o}}$ " from the blade element lying between " $\mathrm{r}$ " and " $\mathrm{r}+\mathrm{dr}$ " is given by:

$$
\begin{gathered}
\frac{d p_{o}}{d r}=\frac{j n B^{2} \Omega}{2 \pi R c\left(1-M_{F} \cos \theta\right)} J_{ \pm n B}\left[\frac{n B \Omega r \sin \theta}{c\left(1-M_{F} \cos \theta\right)}\right] \\
\exp \left[j\left[\frac{\bar{k}(R+\Delta x \cos \theta)}{\left(1-M_{F} \cos \theta\right)} \pm n B\left(\phi-\frac{\pi}{2}\right) \mp n B \alpha_{1}(r)-n B \Omega t\right]\right] \\
{\left[\frac{f_{z o} \cos \theta}{\left(1-M_{F} \cos \theta\right)} \mp \frac{c f_{\phi o}}{\Omega r}+\frac{j n B \rho_{o} c \Omega h l}{\left(1-M_{F} \cos \theta\right)^{2}}\right]}
\end{gathered}
$$

In Equation (1), the far field noise is expressed in terms of $\mathrm{R}, \theta$ (the coordinates of the observer relative to the "retarded" propeller disk location). The relationship between these coordinates and $\mathrm{R}_{\mathrm{c}}, \theta_{\mathrm{c}}$ (the coordinates of the observer relative to the current propeller disk location) is shown in Figures 2 and 3. In Equation (1), wherever two signs appear, the upper sign refers to counterclockwise rotor rotation, and the lower sign to clockwise rotation (forward looking aft).

Now consider the noise due to unsteady loading. Let the unsteady loading per blade per unit spanwise length in a frame of reference fixed with a rotating blade be expressed in the form: 


$$
\text { Unsteady z component }=\sum_{k=1}^{\infty} f_{z k} \cos (k \Omega t)
$$

and

$$
\text { Unsteady } \phi \text { component }=\sum_{k=1}^{\infty} f_{\phi k} \cos (k \Omega t)
$$

Both $\mathrm{f}_{\mathrm{zk}}$ and $\mathrm{f}_{\phi \mathrm{k}}$ are positive functions of "r". Also, let $\beta_{\mathrm{zk}}(\mathrm{r})$ and $\beta_{\phi k}(\mathrm{r})$ denote the azimuthal angles in the coordinate system of Figure 1 where these unsteady components achieve their maximum positive values. Then, unsteady loading noise from blade elements lying between " $r$ " and " $r+d r$ " contributes to the far field pressure a quantity $\mathrm{dp}_{\mathbf{k}} / \mathrm{dr}$, given by the sum of:

$$
\left(\frac{d p_{k}}{d r}\right)_{+} \text {and }\left(\frac{d p_{k}}{d r}\right)
$$

where

$$
\begin{gathered}
\left(\frac{d p_{k}}{d r}\right]_{+}=\frac{j n B^{2} \Omega}{4 \pi R c\left(1-M_{F} \cos \theta\right)} \cdot J_{( \pm n B+k)}\left[\frac{n B \Omega r \sin \theta}{c\left(1-M_{F} \cos \theta\right)}\right] \\
\exp \left[j\left[\frac{\bar{k}(R+\Delta x \cos \theta)}{\left(1-M_{F} \cos \theta\right)}+( \pm n B+k)\left(\phi-\frac{\pi}{2}\right] \mp n B \alpha_{1}(r)-n B \Omega t\right]\right. \\
{\left[\frac{\exp \left(-j k \beta_{z k}\right) f_{z k} \cos \theta}{\left(1-M_{F} \cos \theta\right)} \mp \frac{c \exp \left(-j k \beta_{\phi k}\right) f_{\phi k}(n B \pm k)}{n B \Omega r}\right]}
\end{gathered}
$$

and

$$
\begin{gathered}
\left(\frac{d p_{k}}{d r}\right]_{.}=\frac{j n B^{2} \Omega}{4 \pi R c\left(1-M_{F} \cos \theta\right)} \cdot J_{( \pm n B-k)}\left[\frac{n B \Omega r \sin \theta}{c\left(1-M_{F} \cos \theta\right)}\right] \\
\exp \left[j\left[\frac{\bar{k}(R+\Delta x \cos \theta)}{\left(1-M_{F} \cos \theta\right)}+( \pm n B-k)\left(\phi-\frac{\pi}{2}\right) \mp n B \alpha_{1}(r)-n B \Omega t\right]\right] \\
{\left[\frac{\exp \left(j k \beta_{z k}\right) f_{z k} \cos \theta}{\left(1-M_{F} \cos \theta\right)}+\frac{c \exp \left(j k \beta_{\phi k}\right) f_{\phi k}}{n B \Omega}(n B \mp k)\right]}
\end{gathered}
$$


The total far field acoustic pressure can now be computed as:

$$
p=\int_{r_{h}}^{r_{t}}\left[\frac{d p_{o}}{d r}+\sum_{k=1}^{\infty}\left(\frac{d p_{k}}{d r}\right)_{+}+\sum_{k=1}^{\infty}\left(\frac{d p_{k}}{d r}\right)\right] d r
$$

where $r_{h}$ and $r_{t}$ denote the inner and outer radii of the propeller disk, respectively, and where "p" is a complex number whose amplitude gives the amplitude of the far field pressure in the nth harmonic of blade passing frequency.

The above formulation could, perhaps, be expressed more concisely with more extensive use of complex notation. However, it was felt preferable to restrict consideration to positive $\mathrm{n}$ and $\mathrm{k}$ and, also, to consider both clockwise and counterclockwise rotating propellers. These interests, and the desire to be accurate concerning phase relations, have led to the above formulation.

The theory needed to compute the steady axial and tangential forces for a SRP is based on that of Glawert (1963). The formulation is based on incompressible flow theory and the inputs needed are: relative radius ( $r / R$ ) of interest, solidity (chord/transverse spacing) at this radius of interest, number of blades, specification of the section lift and drag coefficients as functions of angle-of-attack (including the orientation of the reference line with reference to which the angle-of-attack is specified), and the speed ratio, $\lambda=U / \Omega$. The version used for the work described herein incorporates the Prandtl tip loss factor.

For the CRP case, an extension of Glauert's theory by Lock (1941) with inclusion of an approximate accounting of slipstream contraction is used.

In order to ensure that lift and drag coefficients as functions of angle of attack are realistic, these relations were deduced from performance maps for the relevant propellers by inverting the Glauert/Lock theories at a representative span location such as $70 \%$ span. This however entails several additional assumptions such as that for the flow per unit area and work addition per unit mass of fluid are uniform. We assume that the coefficient relations deduced in this manner for the $70 \%$ 
span location are valid for all spanwise locations.

To determine the unsteady forces, in this study, we have used a quasi-steady approximation based on the Glauert/Lock theories to deduce the unsteady forces. The angle of attack induces a low frequency modulation of the blade loading in a frame of reference fixed with the rotor blades and hence a quasi-steady approximation should be adequate. The quasi-steady approximation for SRP's and CRP's is outlined in Appendix 1.

A fairly complete theory data comparison with data from Block (1984) based on the theory of this section can be found in Whitfield (1989). In Block (1984), the tests were carried out on a four bladed SRP designated as an SR2. Some sample results from Whitfield (1989) are reproduced herein.

Figure 6 shows results for the axisymmetric sound field at zero angle of attack at a tip speed of 443 fps. In Figure 7 a theory-data comparison is shown for the change in noise due to angle of attack in the plane of rotation. Figures 8 and 9 show similar results for the same propeller at a higher tip speed of $745 \mathrm{fps}$. It is a characteristic of the theory outlined in this section that, in the plane of rotation, the acoustic factor for BPF noise determining the ratio of unsteady loading to steady loading effects scales as " $J_{B-1}\left(B M_{t}\right) / J_{B}\left(B M_{t}\right)$ ". Since this ratio diminishes as " $\mathrm{M}_{\mathrm{t}}$ " (wheel tip Mach number) increases through subsonic values, it is not surprising that smaller angle of attack effects are predicted for a higher tip speed (Figure 9 versus Figure 7).

The results in Figures 7 and 9 are encouraging considering the data scatter though it is arguable that a systematic underprediction of the magnitude of the noise decrease is evident in Figure 9.

In Figures 10(a) and 10(b), we show the results of applying the theory of the present section to angle of attack effects measured by Woodward (1987b). These measurements are on a $11 \times 9$ bladed CRP spinning at approximately $800 \mathrm{fps}$ in a Mach 0.2 airstream. The theory-data comparisons for the angle of attack effect on the BPF noise of the front rotor indicate a severe underprediction of the measured effect. 
The key difference between the propellers tested by Block (1984) and Woodward (1987b) is that the latter propellers operate with substantially higher steady loading (lift coefficients are higher by a factor of 2-3) and the number of blades involved in the Woodward experiments (11 and 9) is much greater than the four blades used in the Block experiment.

In the course of analyzing the disappointing results of Figure 10(a) and 10(b) (an exhaustive theory-data comparison based on the results of the present section was carried out with all the BPF data available in the Woodward experiments with results typically as shown in Figure 10) the author was made aware by colleagues at the NASA Lewis Research Center that presently unpublished research has yielded similar gross underprediction of the measured azimuthal variation of BPF noise with angle of attack. Estimates of the unsteady force levels (derived herein by a quasi-steady approximation) are of course of interest in the context of forced vibration of the propeller blades and conversations with colleagues in that discipline indicate that the unsteady force estimates provided by the present procedure for the angle of attack effect are reasonable. It also seems necessary to contend with the observation that the theory of the present section appears to perform adequately in explaining the Block (1984) experiments with lightly loaded, four bladed propellers.

The experience cited above has led to a re-examination of a basic premise of the present development; namely, that the steady loading/thickness noise itself is unaffected by angle of attack. As the discussion of the next section will show, this premise appears incorrect. An approximate accounting of the effect of angle of attack on steady loading/thickness noise is carried and a very substantial improvement is now evident in terms of the Woodward (1987b) studies. The development of the next section will also elucidate why this effect is relatively unimportant for lightly loaded, low tip speed propellers with a few number of blades as were involved in the Block (1984) experiments. 


\section{The Alteration of Steady Loading/Thickness Noise Due to Angle of Attack}

The physical mechanism which causes the steady loading/thickness noise of a propeller to be fundamentally non axially symmetric (i.e. in both amplitude and phase) when the propeller is at angle of attack is quite simple and can be explained in terms of Figure 11.

In this figure we show a steady source executing a circular motion as would be characteristic of steady sources rotating with the blade. Also shown in the figure is the uniform upwash provided by the cross flow due to angle of attack. Now the radiative efficiency of a source depends on the relative velocity between the source and the fluid surrounding it and as Figure 11 shows this relative velocity (and hence the radiative efficiency) is modulated in a non axisymmetric fashion due to the cross flow. This effect has been neglected in prior analyses.

To capture this effect, we proceed as follows. Including the cross flow (designated as " $\mathrm{M}$ " in Figure 12), the acoustic pressure due to steady loading satisfies:

$$
p_{x x}\left(1-M_{x}^{2}\right)+p_{y y}\left(1-M_{y}^{2}\right)+p_{z z}+2 j k M_{x} p_{x}+2 j k M_{y} p_{y}-2 M_{x} M_{y} p_{x y}+k^{2} p=\left(\frac{\partial f_{x}}{\partial x}+\frac{1}{r} \frac{\partial f_{\phi}}{\partial \phi}\right)
$$

where $\mathrm{k}=\omega / \mathrm{c}$, all quantities have a time dependence as $\exp (-\mathrm{j} \omega t)$, "c" is the speed of sound, $M_{X}$ and $M_{y}$ are axial and cross flow Mach numbers, " $f_{x}, f_{\phi}$ " are the force terms and $j=\sqrt{-1}$ and $p_{x x}$ denotes $\partial^{2} p / \partial x^{2}$, etc. In the following, $M_{X}$ will be denoted by $M$.

Let

$$
\begin{aligned}
& f_{x}=f_{x o} \exp (j m \phi) \delta(x) \delta(r-a) \\
& f_{\phi}=f_{\phi o} \exp (j m \phi) \delta(x) \delta(r-a)
\end{aligned}
$$

where " $\phi$ " is the azimuthal coordinate. In the present study, it has been possible to solve the above problem only to $\mathrm{O}\left(\mathrm{M}_{\mathrm{y}}\right)$.

Define the axial Fourier transform pair: 


$$
P=\int_{-\infty}^{\infty} p \exp (-j \alpha x) d x
$$

and

$$
p=\frac{1}{2 \pi} \int_{-\infty}^{\infty} P \exp (j \alpha x) d \alpha
$$

Then to $O\left(M_{y}\right)$, P satisfies:

$$
P_{y y}+P_{z z}+2 j M_{y} P_{y}(k-\alpha M)+\left[(k-\alpha M)^{2}-\alpha^{2}\right] P=j\left[\alpha f_{x o}+\left(\frac{m}{a}\right) f_{\phi o}\right] \delta(r-a) e^{j m \phi}
$$

Now let $\bar{P}=P \exp \left[j y M_{y}(k-x M)\right]$

Then to $\mathrm{O}\left(\mathrm{M}_{\mathrm{y}}\right), \bar{P}$ satisfies:

$$
\begin{gathered}
\bar{P}_{y y}+\bar{P}_{z z}+\left[(k-\alpha M)^{2}-\alpha^{2}\right] \bar{P}=j\left[\alpha f_{x o}+m f_{\phi o} / a\right] \\
\delta(r-a) e^{j m \phi}\left[1+M_{y}(k-\alpha M) a\left(\frac{e^{j \phi}-e^{-j \phi}}{2}\right)\right]
\end{gathered}
$$

Let

$$
\gamma=\sqrt{(k-\alpha M)^{2}-\alpha^{2}}
$$

Then

$$
\begin{gathered}
P=\frac{\pi}{2}\left[(\alpha a) f_{x o}+m f_{\phi o}\right] \exp \left[-j M_{y} y(k-\alpha M)\right] \\
{\left[e^{j m \phi} J_{m}(\gamma a) H_{m}^{(1)}(\gamma)+\frac{M_{y}(k-\alpha M) a}{2} e^{j(m+1) \phi}\right.} \\
\left.J_{m+1}(\gamma a) H_{m+1}^{(1)}(\gamma r)-\frac{M_{y}(k-\alpha M) a}{2} e^{j(m-1) \phi} J_{m-1}(\gamma a) H_{m-1}^{(1)}(\gamma r)\right]
\end{gathered}
$$

We can now set $\mathrm{x}=-\mathrm{R}_{\mathrm{c}} \cos \theta_{\mathrm{c}^{\prime}}, \mathrm{r}=\mathrm{R}_{\mathrm{c}} \sin \theta_{\mathrm{c}}$ and $\mathrm{y}=\mathrm{R}_{\mathrm{c}} \sin \theta_{\mathrm{c}} \sin \phi$ and carry out an asymptotic 
evaluation of "p" for large $R_{c}$. The full details of this asymptotic evaluation are not necessary and it suffices to note the following. Firstly in the evaluation of the " $\mathrm{m}$ " azimuthal mode in the above there are $O\left(M_{y}\right)$ shifts in the point of stationary phase (relative to the case $M_{y}=0$ ). This shift of the point of stationary phase is illustrated in Figure 13, in terms of the resulting new relation between current and retarded coordinates. It is reiterated that Figure 13 is valid only to $O\left(M_{y}\right)$. Note that the relation between current and retarded coordinates itself now is non axially symmetric i.e. it depends on " $\phi$ ". However this detail is far less significant than the appearance of new azimuthal modes of order $(\mathrm{m}-1)$ and $(m+1)$ in the equation above. For the " $m-1 "$ and " $m+1 "$ modes we can use the point of stationary phase as for "M$=O$ " since these are $O\left(M_{y}\right)$ contributions and hence relative to the " $m$ " mode, these contribute as:

$$
\begin{gathered}
m-1: \frac{-\exp \left[-j(\phi-\pi / 2] M_{y}(k a)\right.}{2(1-M \cos \theta)} J_{m-1}\left(\frac{k a \sin \theta}{(1-M \cos \theta)}\right) \\
m+1: \frac{\exp [j(\phi-\pi / 2)] M_{y}(k a)}{2(1-M \cos \theta)} J_{m+1}\left(\frac{k a \sin \theta}{(1-M \cos \theta)}\right)
\end{gathered}
$$

where the relation between " $\theta_{c}$ " and retarded angular coordinate " $\theta$ " is as shown in Figure 3. Clearly the "m-1" term contributes much more significantly than the " $m+1$ " term. A virtually identical treatment applies to thickness noise. Note that "ka $=n M_{t}$ " where " $n$ " is harmonic of BPF noise, "B = number of blades" and " $M_{t}=$ wheel tip Mach number" at radius "a".

The modifications to equation (1) to $O\left(M_{y}\right)$ will now be written out. In order to clearly motivate this development, this procedure will be carried out step-by-step. Firstly we reconsider and rewrite equation (1). Introduce the following symbols. 


$\begin{array}{lll}\text { Symbol } & \text { Value } & \text { Significance } \\ \text { prmlt } & j n B^{2} \Omega /(2 \pi C) & \text { premultiplier } \\ \text { camp } & 1 /(1-\mathrm{M} \cos \theta) & \begin{array}{l}\text { convective } \\ \text { amplification factor }\end{array} \\ \text { arg } & n B \Omega r \sin (\theta)(\operatorname{camp}) / \mathrm{c} & \begin{array}{l}\text { argument of } \\ \text { Bessel function }\end{array} \\ & & \\ \text { pht } & \exp \left[j\left[\frac{\bar{k}(R+\Delta x \cos \theta)}{(1-M \cos \theta)} \pm n B\left(\theta-\frac{\pi}{2}\right)\right.\right. & \text { phase } \\ & \left.\left.\mp n B \alpha_{1}(r)-n B \Omega t\right]\right] & \text { term } \\ \text { tht } & \mathrm{jnB} \rho_{o} c \Omega h l & \begin{array}{l}\text { thickness } \\ \text { term }\end{array}\end{array}$

With the above symbols, the present form of equation (1) can be written as:

$$
\begin{gathered}
\quad \frac{d p_{o}}{d r}=\frac{(p r m l t)(p h t)}{R}\left(f_{z o} \cos (\theta)(c a m p)^{2} J_{ \pm n B}(a r g)\right. \\
\left.\mp \frac{c f_{\phi o}(c a m p)}{\Omega r} J_{ \pm n B}(a r g)+(t h t)(c a m p)^{3} J_{ \pm n B}(a r g)\right)
\end{gathered}
$$

When effects on steady loading/thickness noise to $\mathrm{O}\left(\mathrm{M}_{\mathrm{y}}\right)$ are calculated, the following new symbols are introduced.

i. aat (for "angle of attack" term):

$$
\begin{aligned}
& (\text { aat })=\left(n B \cap r M_{y}(\text { camp }) / 2 c\right) \\
& \left(\exp (j(\phi-\pi / 2)) J_{ \pm n B+1}(\text { arg })-\right. \\
& \left.\exp (-j(\phi-\pi / 2)) J_{ \pm n B-1}(\text { arg })\right)
\end{aligned}
$$

ii. $\quad M_{y \phi}=M_{y} \sin \phi$ (component of cross flow Mach number in " $\phi$ " direction)

iii. $\Delta \theta=M_{y \phi}(M-\cos \theta) /(1-M \cos \theta)$ (change in emission angle to $\mathrm{O}\left(\mathrm{M}_{\mathrm{y}}\right)$ due to cross flow for fixed

$$
\left.\mathrm{R}_{\mathrm{c}}, \theta_{\mathrm{c}}\right)
$$

iv. $\cos (\psi)=\cos \theta-\Delta \theta \sin \theta$

$$
\sin (\psi)=\sin \theta+\Delta \theta \cos \theta
$$

( $" \psi$ " is the new emission angle to $O\left(\mathrm{M}_{\mathrm{y}}\right)$ for fixed $\mathrm{R}_{\mathrm{c},}{ }_{\mathrm{c}}$ ) 
v. $\mathrm{R}_{\mathrm{n}}=\mathrm{R}\left(1-M_{y \phi} /(1-M \cos \theta)\right)$

( $R_{n}$ is the new distance of observer from center of retarded propeller to $O\left(M_{y}\right)$ for fixed $R_{c^{\prime}}$ $\left.\theta_{c}\right)$

vi. campn (for new "convective amplification factor") $=1 /\left(1-M \cos \psi+M_{y \phi} \sin \theta\right)$

vii. $\operatorname{argn}\left(\right.$ for new argument of Bessel functions) $=n B \Omega\left(\sin \psi+M_{y \phi}\right)($ campn $) / c$

Then the result analogous to equation (1) including the crossflow effect to $O\left(M_{y}\right)$ is:

$$
\begin{gathered}
\frac{d p_{o}}{d r}=(\text { prmlt })(p h t)\left[f _ { z o } \left(\cos \psi(\text { campn })^{2}\right.\right. \\
\left.J_{ \pm n B}(\text { argn }) / R_{n}+(a a t) \cos (\theta)(\text { camp })^{2} / R\right) \\
\mp \frac{c f_{\phi o}}{\Omega r}\left(\frac{(\text { campn }) J_{ \pm n B}(a r g n)}{R_{n}}+\frac{(a a t)(\text { camp })}{R}\right) \\
\left.+(\text { tht })\left((\text { campn })^{3} J_{ \pm n B}(\text { argn }) / R_{n}+(a a t)(\text { camp })^{3} / R\right)\right]
\end{gathered}
$$




\section{Revised Theory-Data Comparisons and Discussion}

The results presented in this section use the revised acoustic theory for steady loading/thickness noise and the old acoustic theory (wherein the only mean flow effect dealt with is a uniform, axial flow) for the unsteady loading/thickness noise. This approach is consistent with the idea that at present only an $\mathrm{O}\left(\mathrm{M}_{\mathrm{y}}\right)$ acoustic theory has been developed where " $\mathrm{M}_{\mathrm{y}}$ " is the crossflow Mach number due to angle of attack. All " $\theta$ " coordinates referred to in Figures 14-21 are current coordinates (as opposed to retarded coordinates). All predictions are for the fundamental BPF tone of the relevant propeller and are for the noise change due to angle of attack.

In Figures 14-15, using the new acoustic theory, we show theory-data comparisons for the Block (1984) data. It can be seen that the comparisons are at least as good as shown in Figures 7 and 9. The new effect discussed in the previous section scales as "nBM$t$ " and pertains only to steady loading/thickness noise. Thus it is not surprising that the new effect is not very consequential for a lightly loaded, four bladed propeller such as the SR-2 used in the Block (1984) experiments.

In Figures 16-21 we show the theory data comparisons for the CRP data of Woodward (1987b). Several comments concerning these theory-data comparisons are noted below.

1. In cases where the measured " $\theta$ " is not close to a multiple of 10 degrees, predictions for multiples of 10 degrees bracketing the measured " $\theta$ " are both compared with the data.

2. Figures $16(a),(b)$ may be compared to Figures $10(a),(b)$ (the data shown in these figures are the same). A dramatic improvement is now evident with the revised acoustic theory in terms of the substantial under prediction of the old acoustic theory of the angle of attack effect.

3. Figures 17 and 19 indicate that angle of attack effects on the rear rotor noise are still significantly underpredicted. The rear rotor in Woodward (1987) has fewer blades (9 versus 11) than the front rotor and hence the predicted magnitude of the new effect (recall the "nBM scaling law for the new effect) is correspondingly smaller. Also as far as unsteady effects are 
concerned, the steady and quasi-steady CRP theory developed herein predicts a "smoothing" effect of the front rotor in terms of the effect of inflow distortions on the rear rotor and hence the unsteady effects on the rear rotor are also predicted to be smaller than on the front rotor. At any rate the reasons for underprediction of effects on the rear rotor are not clear.

4. Finally if we examine figures $18(a),(b), 19$ (as a typical case), it would appear that the maxima and minima of the data appear at larger " $\phi$ " with respect to the theory for the front rotor and vice-versa for the rear rotor. For the CRP used in Woodward (1987b), the front rotor spins in the direction of decreasing " $\phi$ " (clockwise forward looking aft). It may be surmised that when an elongated centerbody such as used in Woodward (1987b) is placed at an angle of attack to the freestream and the propellers are placed aft of the maximum diameter location of the centerbody, the propeller blades could experience a once per rev distortion of incoming axial velocity such that this velocity is diminished on top and increased on the bottom. In Figures 20(a),(b) and 21 , a calculation was carried out assuming the presence of such an axial velocity distortion of magnitude $10 \%$ of the freestream velocity. Comparing these results with figures $18(\mathrm{a}),(\mathrm{b})$ and 19 , clearly a shift is obtained of the theory which is in the right direction relative to the data. The purpose of this exercise is to indicate that small distortions of axial velocity additional to the tangential velocity distortion created by angle of attack can cause significant shifts of the predictions in the " $\phi$ " direction even if the magnitudes are not much affected.

A complete version of theory-data comparisons with all of the data obtained by Woodward (1987b) will be published in a NASA contractor's report to follow. 


\section{Concluding remarks}

In this study an approach based on steady and quasi-steady aerodynamics of a propeller has been developed to predict both the steady loading/thickness BPF noise and the unsteady loading/thickness BPF noise developed by a propeller operating at angle of attack. Originally a conventional acoustic theory was used in which the only mean flow effect accounted for is a uniform axial flow. Application of this approach to data of Woodward (1987b) indicated a severe underestimation of the magnitude of the effect of angle of attack on BPF noise from a CRP. A new idea has been developed in this paper based on the notion that the small crossflow due to angle of attack (designated in Mach number as "M non uniform). This effect may be far more important than unsteady loading/thickness noise for heavily loaded, high tip Mach number propellers with a large number of blades. This effect has been calculated only to $O\left(M_{y}\right)$ in this study. Revised theory-data comparisons indicate that the underestimation problem is substantially resolved especially for BPF noise from the front rotor though underestimation of the magnitude of noise decreases due to angle of attack for BPF noise from the rear rotor is still in evidence. Since the $O\left(M_{y}\right)$ effect has itself proved to be large, it is suggested that research is needed to calculate the acoustic impact of the crossflow more fully. 


\section{Acknowledgements}

Sincere thanks are expressed to P.J. Block, R.P. Woodward and D.M. Hill for their assistance in providing access to original data. This study was financially supported by a contract from NASA, Lewis Research Center, Cleveland, Ohio (USA). 


\section{$\underline{\text { Single Rotation Propellers }}$}

Let $u(r, \phi), v(r, \phi)$ denote externally contributed nonuniform axial and tangential velocities incident on the propeller. " $\mathrm{u}$ " is taken positive in the downstream direction and "v" is taken positive in the direction of propeller rotation.

At any radius, the quasi-steady forces (thrust and tangential force) are obtained in the functional form:

$$
F=\Omega^{2} f(\lambda)
$$

where " $\Omega$ " denotes the propeller rotational velocity in radians per second, and " $\lambda$ " the speed ratio

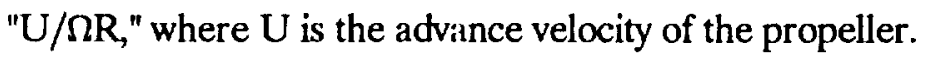

The change in the force, $F$, due to $u$ and $v$ can be obtained as follows.

In the quasi-steady approximation, changes in $\Omega$ and $\lambda$ due to $u$ and $v$, will be:

$$
\begin{gathered}
\delta \Omega=-\frac{v}{r} \\
\delta \lambda=\lambda\left(\frac{u}{U}+\frac{v}{\Omega r}\right)
\end{gathered}
$$

The resulting change in $\mathrm{F}$ can be written:

$$
\begin{gathered}
\delta F=2 \Omega f(\lambda) \delta \Omega+\Omega^{2}\left(\frac{\partial f}{\partial \lambda}\right) \delta \lambda \\
=\left[-2 F+\lambda\left(\frac{\partial F}{\partial \lambda}\right)\right] \frac{v}{\Omega r}+\left(\frac{\partial F}{\partial \lambda}\right) \frac{u}{\Omega R}
\end{gathered}
$$

Thus, if $u, v$ are nondimensionalized by introducing: 


$$
u^{\prime}=\frac{u}{\Omega R} \text { and } v^{\prime}=\frac{v}{\Omega R}
$$

we have:

$$
\delta F=\left(-2 F+\lambda \frac{\partial F}{\partial \lambda}\right) \frac{R}{r} v^{\prime}+\frac{\partial F}{\partial \lambda} u^{\prime}
$$

Now, $\partial F / \partial \lambda$ can be approximated by a central difference formula:

$$
\frac{\partial F}{\partial \lambda} \sim \frac{F(\lambda+\varepsilon)-F(\lambda-\varepsilon)}{2 \varepsilon}
$$

where " $\varepsilon$ " is a small increment. 


\section{Counter Rotation Propellers}

Now, in general, the extension of the steady single rotation propeller theory to the counter rotation case will yield for quantities $\left(Q_{F}\right)$ associated with the front rotor a functional relation of type:

$$
Q_{F}=C_{F} \Omega_{F}^{2} f_{F}(\lambda, S)
$$

where " $\mathrm{C}_{\mathrm{F}}$ " is a suitable constant of proportionality at a given radius. Likewise, for quantities $\left(\mathrm{Q}_{\mathrm{R}}\right)$ associated with the rear rotor we may write:

$$
Q_{R}=C_{R} \Omega_{R}^{2} f_{R}(\lambda, S)
$$

We now wish to compute the variation $\delta Q_{F}, \delta Q_{R}$, due to the extra (u,v) velocity field. We note the following intermediate relations:

$$
\begin{gathered}
\delta \Omega_{F}=-v / r, \delta \Omega_{R}=v / r \\
\delta \lambda=\lambda\left[u / U+v /\left(\Omega_{F}\right)\right] \\
\delta S=-S\left[v /\left(\Omega_{F}\right)+v /\left(\Pi_{R}\right)\right] \\
=-S(1+S) v /\left(\Omega_{F}\right) \\
=-S \cdot(1+S) \cdot \lambda \cdot\left(\frac{R_{p}}{r}\right)(v / U)
\end{gathered}
$$

Thus:

$$
\begin{gathered}
\delta Q_{F}=C_{F}\left[2 \Omega_{F} \delta_{F} f_{F}+\Omega_{F}^{2}\left(\frac{\partial f_{F}}{\partial \lambda}\right) \delta \lambda+\Omega_{F}^{2}\left(\frac{\partial f_{F}}{\partial S}\right) \delta S\right] \\
=C_{F} \Omega_{F}^{2}\left[\lambda \cdot\left(\frac{\partial f_{F}}{\partial \lambda}\right) \cdot\left(\frac{u}{U}\right)+\left[-2 \cdot \lambda \cdot\left(\frac{R_{p}}{r}\right) f_{F}+\lambda^{2} \cdot\left(\frac{R_{p}}{r}\right) \cdot\left(\frac{\partial f_{F}}{\partial \lambda}\right)-S \cdot(1+S) \cdot \lambda \cdot\left(\frac{R_{p}}{r}\right)\left(\frac{\partial f_{F}}{\partial S}\right)\right](v / U)\right]
\end{gathered}
$$

Likewise:

$$
\begin{gathered}
\delta Q_{R}=C_{R}\left[2 \cdot \Omega_{R} \cdot \delta \Omega_{R} \cdot f_{R}+\Omega_{R}^{2} \cdot\left(\frac{\partial f_{R}}{\partial \lambda}\right) \cdot \delta \lambda+\Omega_{R}^{2}\left(\frac{\partial f_{R}}{\partial S}\right) \cdot \delta S\right] \\
=C_{R} \Omega_{R}^{2}\left[\lambda \cdot\left(\frac{\partial f_{R}}{\partial \lambda}\right) \cdot\left(\frac{u}{U}\right)+\left[2 \cdot \lambda \cdot S \cdot\left(\frac{R_{p}}{r}\right) \cdot f_{R}+\lambda^{2} \cdot\left(\frac{R_{p}}{r}\right) \cdot\left(\frac{\partial f_{R}}{\partial \lambda}\right)-S \cdot(1+S) \cdot \lambda \cdot\left(\frac{R_{p}}{r}\right)\left(\frac{\partial f_{R}}{\partial S}\right)\right](v / U)\right]
\end{gathered}
$$


These are the relations that are used to determine the fluctuation quantities in the quasi-steady approximation for the counter rotation case. Note that partial derivatives with respect to both " $\lambda$ " and "S" (advance ratio and speed ratio) are now required. These are evaluated by examining steady solutions for the following five pairs of points in the $(\lambda, S)$ space:

$(\lambda, S),(\lambda+0.01, S),(\lambda-0.01, S),(\lambda, S+0.01)$, and $(\lambda, S-0.01)$. 


\section{REFERENCES}

[1] Block, P.J.W., "The Effects of Installation on Single and Counter Rotation Noise," AIAA paper 84-2263, 1984.

[2] Garrick, I.E. and Watkins, C.E., 1954, "A Theoretical Study of the Effect of Forward Speed on the Free Space Sound Pressure Field Around Propellers," N.A.C.A. Rep. No. 1198.

[3] Glauert, H., "Airplane Propellers" in Vol. IV of Aerodynamic Theory edited by W.F. Durand (Dover Edition, 1963).

[4] Gutin, L., 1936, "Über das Schallfeld einer rotierenden Luftschraube", Phys. 2. Sowjet. 9, 5771.

[5] Hanson, D.B., "Noise of Counter Rotation Propellers," AIAA Paper 84-2305, 1984.

[6] Hawkings, D.L., and Lowson, M.V., "Theory of Open Supersonic Rotor Noise," J. Sound Vib., Vol. 36, No. 1, September 8, 1974, pp. 1-20.

[7] Lock, C.N.H., "Interference Velocity for a Close Pair of Contra-rotating Airscrews," R\&M No: 2084, British A.R.C., July 22, 1941.

[8] Whitfield, C.E., Gliebe, P.R., Mani, R., Mungur, P., "High Speed Turboprop Aeroacoustic Study (Single Rotation)" Vol. I: Model Development, NASA CR 182257, May 1989.

[9] Woodward, R.P., "Measured Noise of a Scale Model High Speed Propeller at Simulated Takeoff/Approach Conditions," AIAA 87-0526, 1987(a).

[10] Woodward, R.P., "Noise of a Model High Speed Counterrotation Propeller at Simulated Takeoff/Approach Conditions (F7/A7)", AIAA 87-2657, 1987(b). 


\section{Appendix 2: Notation}

B Number of propeller blades

c Ambient speed of sound

$\mathrm{C}_{\mathrm{F}}, \mathrm{C}_{\mathrm{R}}$ : Constants of proportionality associated with front and rear rotors at a given radius, $\mathrm{r}$

$\mathrm{f}_{\text {zo }} \quad$ Steady (thrust) force per unit spanwise length exerted by each blade in the $\mathrm{z}$ direction (Figure 2$) ; \mathrm{f}_{\mathrm{zo}}$ is a function of radius " $\mathrm{r}$ "

$f_{\phi o} \quad$ Steady (tangential) force per unit spanwise length exerted by each blade in the " $\phi$ " direction (Figure 1); note that $f_{\phi 0}$ is a function of radius and also that, in view of Figure 1, if the sense of rotor rotation is clockwise (forward looking aft), $f_{\phi o}$ will be negative

h Representative airfoil thickness as a function of radius

j $\sqrt{ }-1$

$\mathrm{J} \quad$ Bessel function of the first kind

$\bar{k} \quad$ Wave number, $n B \Omega / c$

$1 \quad$ Airfoil chord as a function of radius

$\mathrm{M}_{\mathrm{F}} \quad$ Flight Mach number

n Harmonic of blade passing frequency of interest $(n \geq 1)$

$\mathrm{p}_{\mathrm{o}} \quad$ Far field acoustic pressure due to steady loading and thickness

r Radius of interest

R Distance of observer from center of retarded propeller disk location (reference is made to Figure 3) 
$R_{c} \quad$ Distance of observer from center of current propeller disk location (Figure 3)

$\mathrm{R}_{\mathrm{p}} \quad$ Propeller radius

S Rotor speed ratio, i.e., $S=\Omega_{f} / \Omega_{R}$

t Time

U: $\quad$ Forward flight speed

u: $\quad$ Perturbation axial velocity component (positive downstream)

v: $\quad$ Perturbation tangential velocity (positive corresponds to " $v$ " in same sense as direction of rotation of forward rotor)

$\phi \quad$ Azimuthal angles as defined in Figure 1 (note that $\phi=0,180$ represent the horizontal direction)

$\lambda: \quad$ Advance ratio based on front rotor, i.e., $\lambda=U /\left(R \Omega_{F}\right)$

$\rho_{\mathrm{o}} \quad$ Ambient air density

$\theta \quad$ "Emission" angle for propeller noise

$\theta_{c} \quad$ "Observer" angle for propeller noise

$\Omega \quad$ Angular velocity of propeller in radians per second

$\Omega_{F}, \Omega_{R}: \quad$ Speeds of rotation of forward and rear rotor 


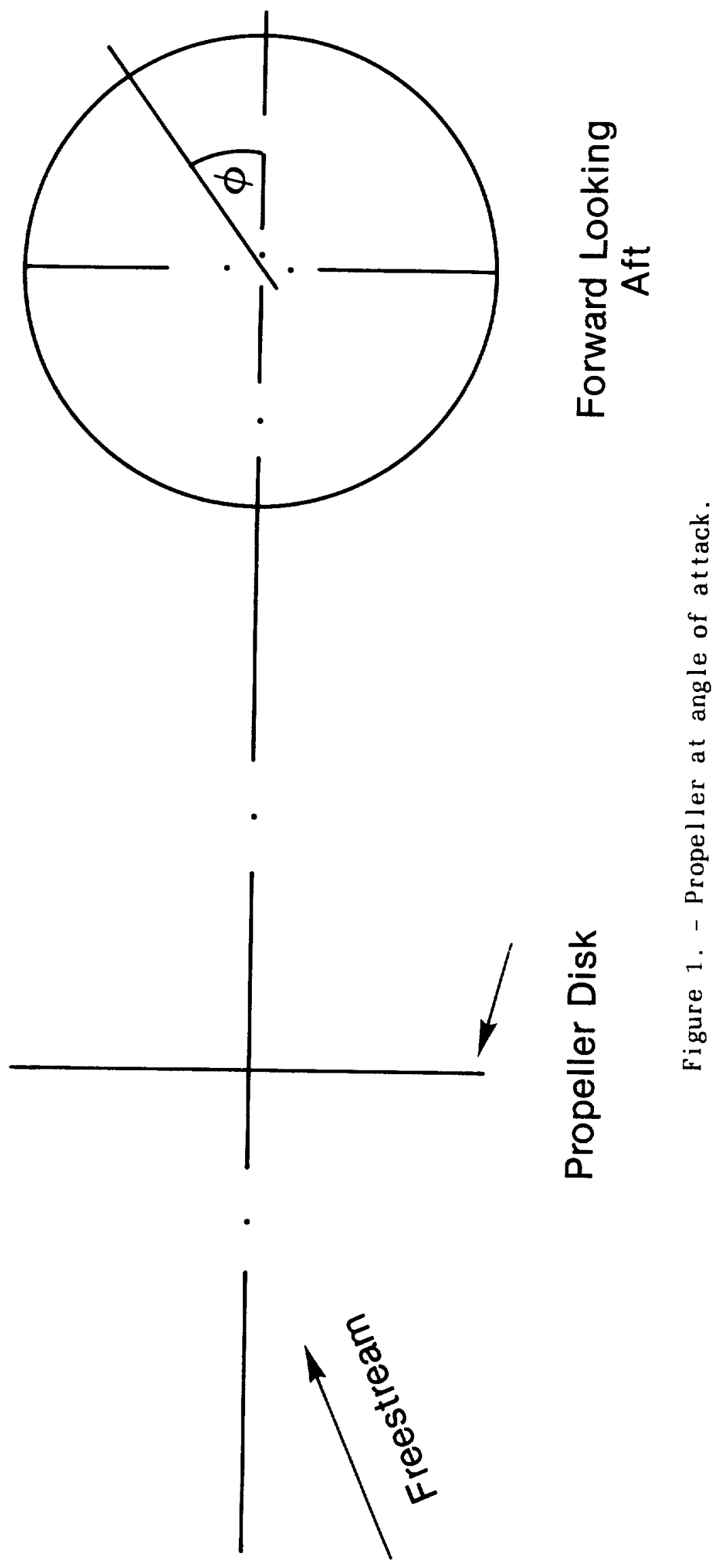




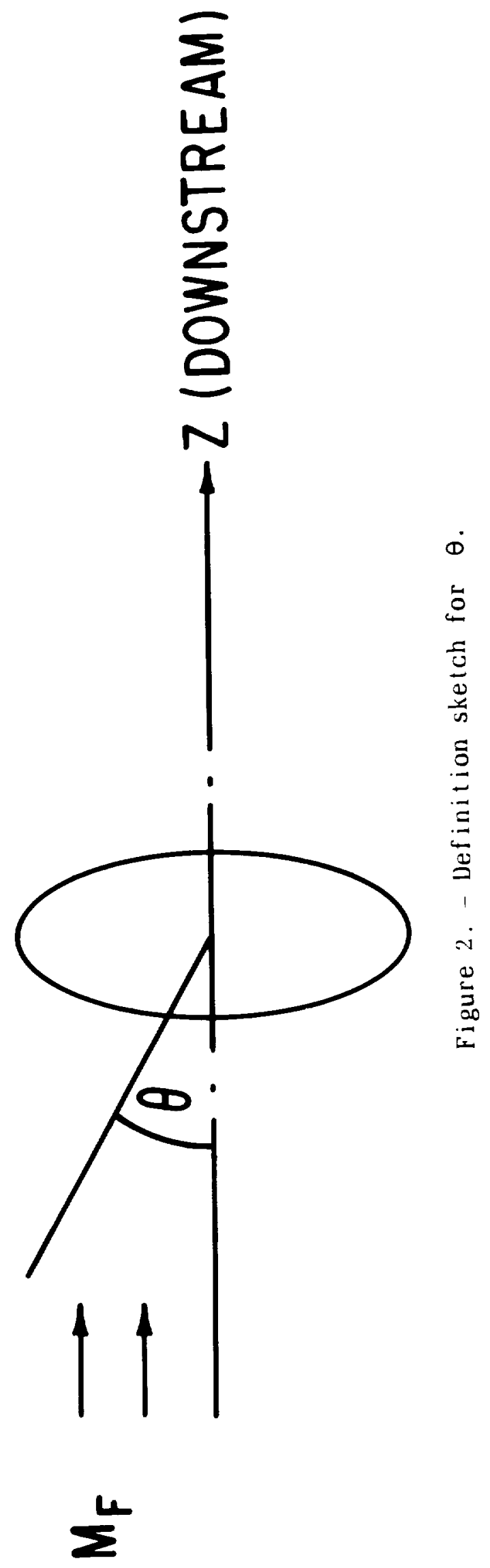




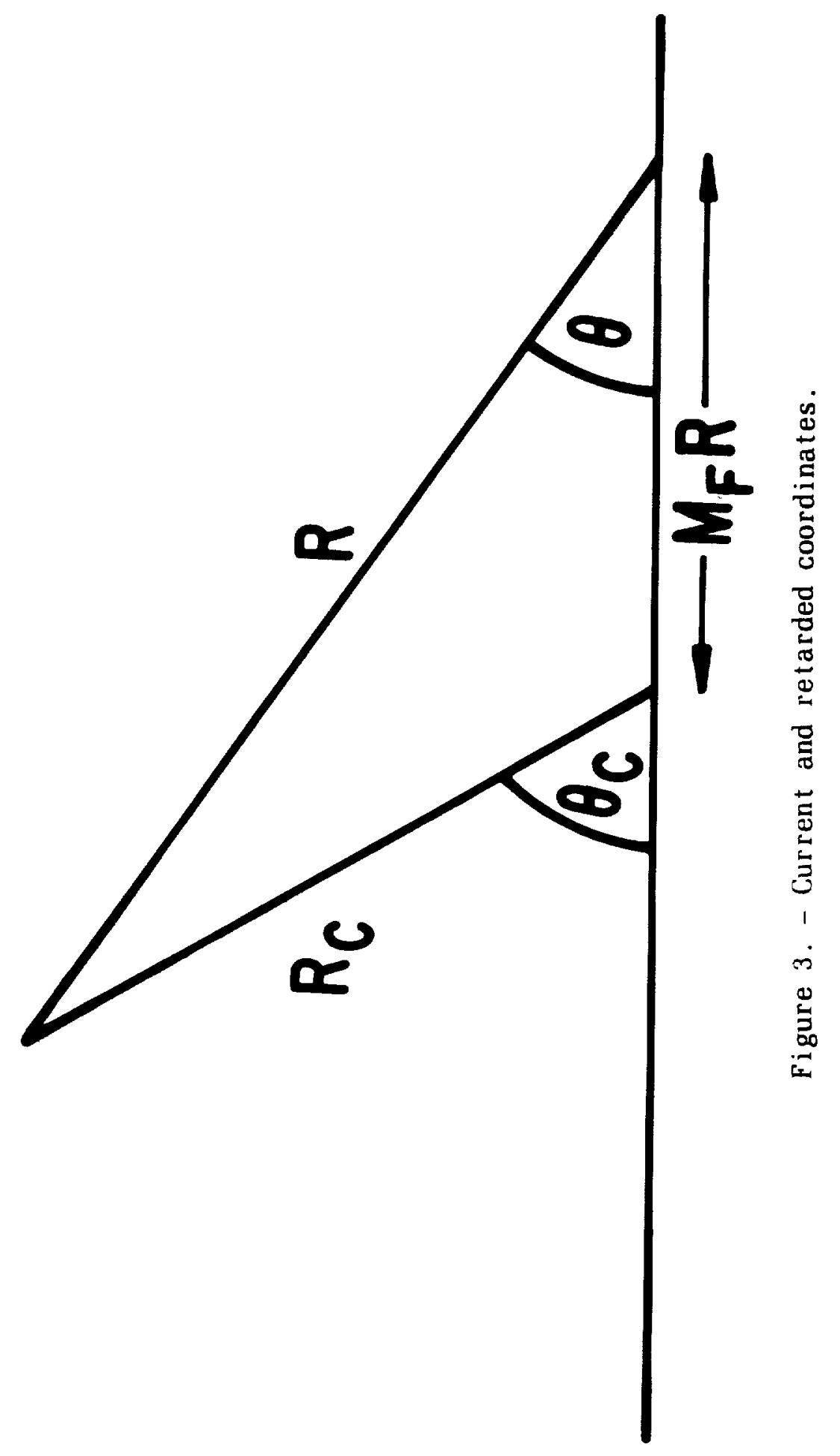




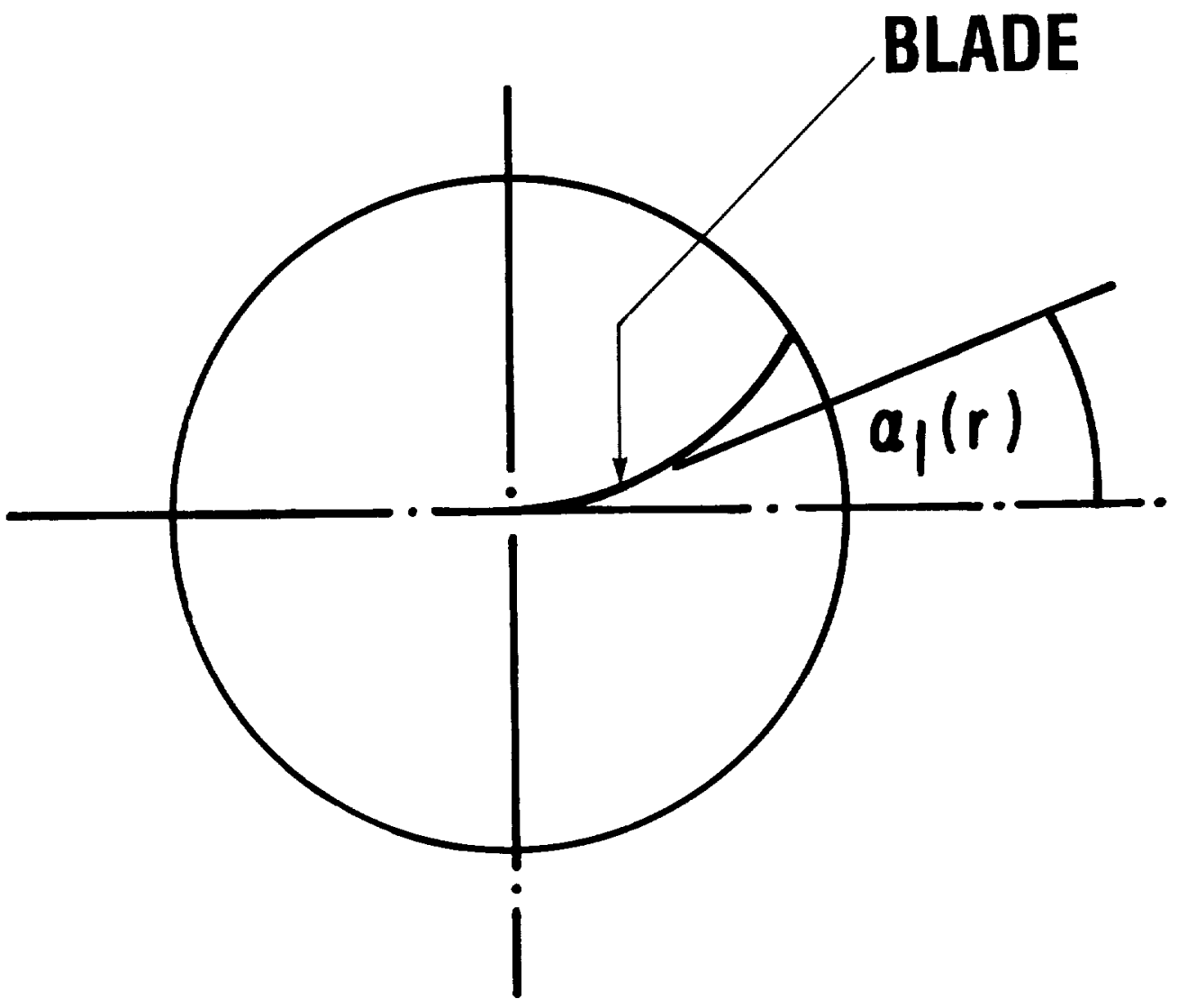

Figure 4. - Definition sketch for radial lean. 


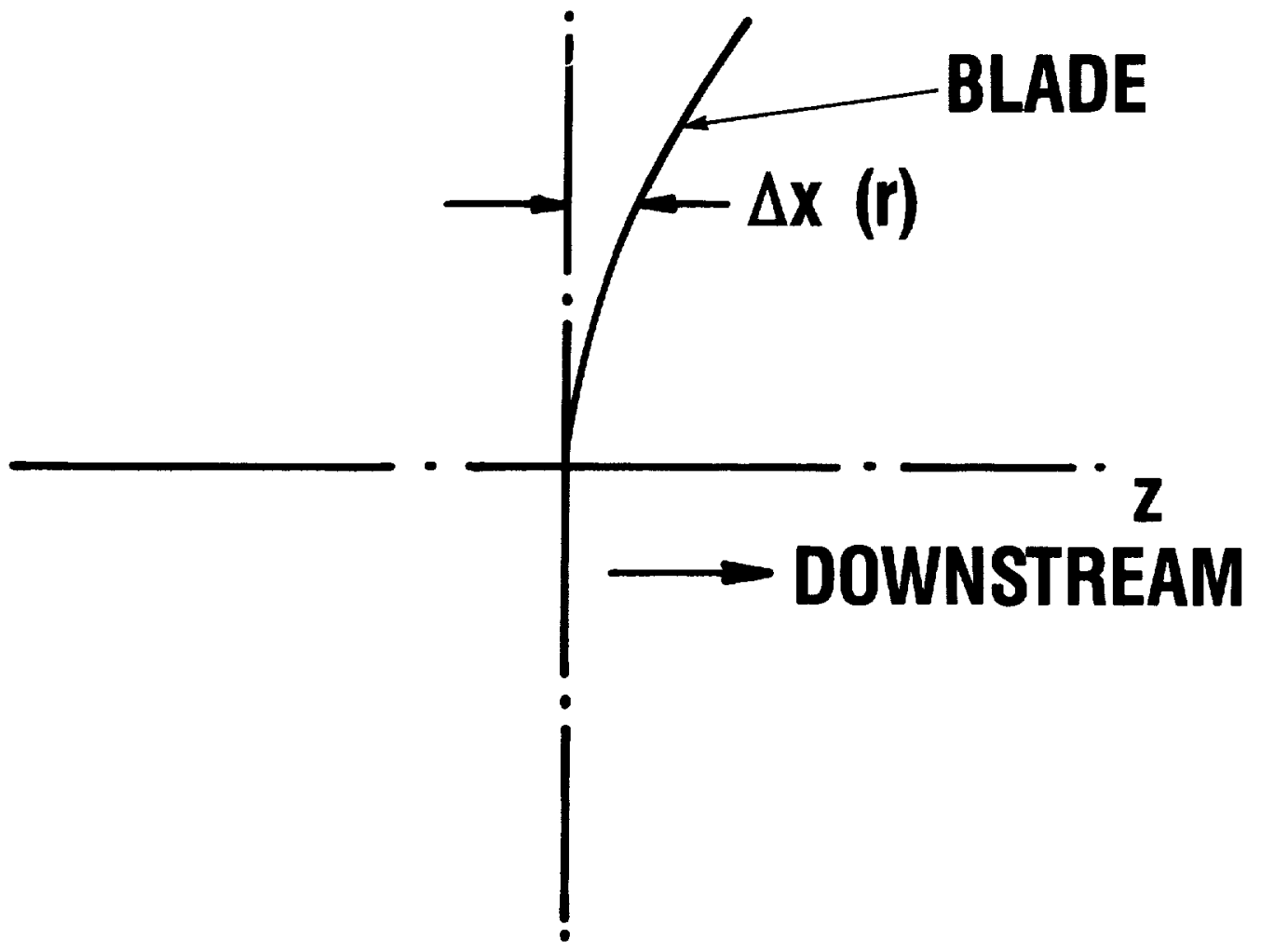

Figure 5. - Definition sketch for axial sweep. 


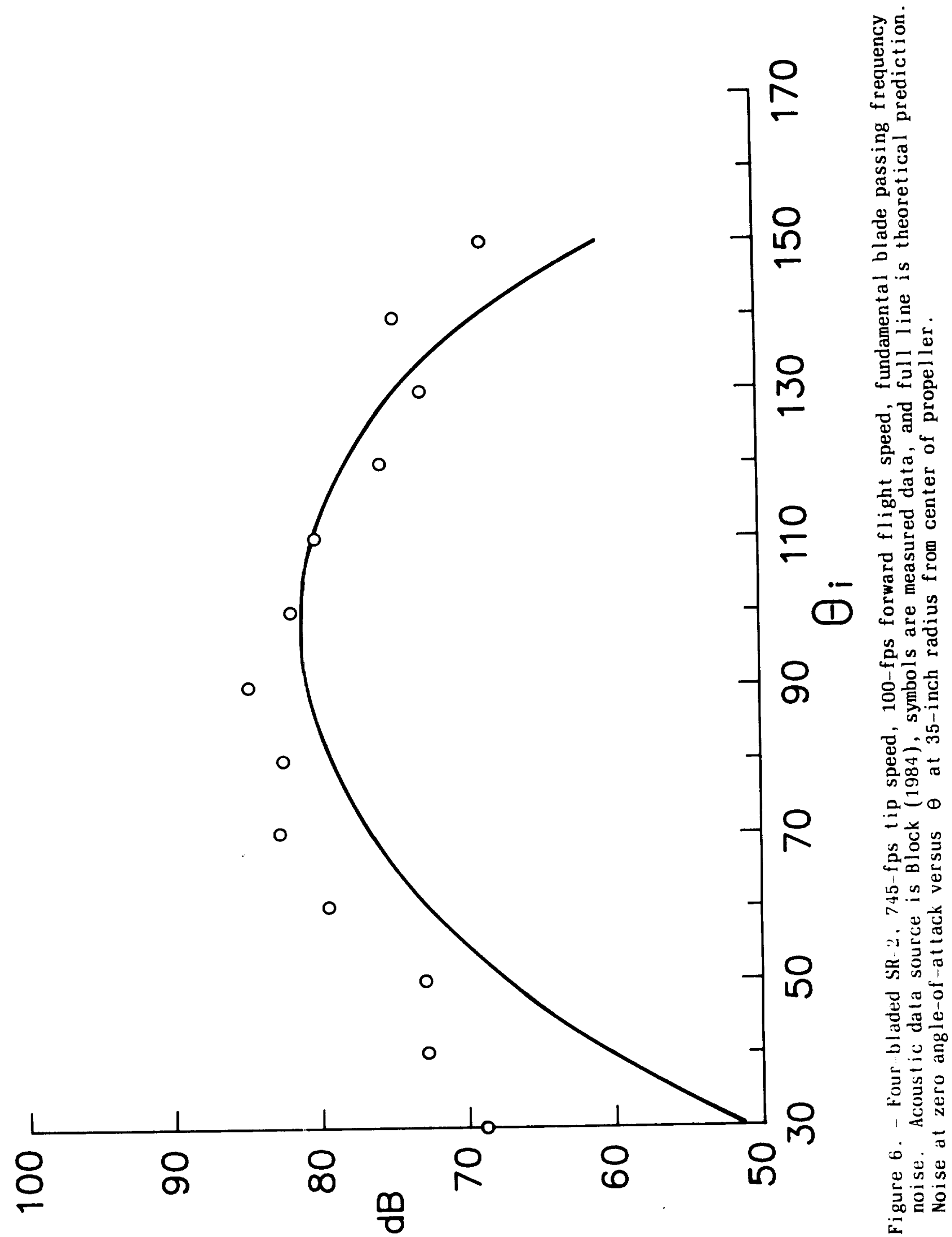




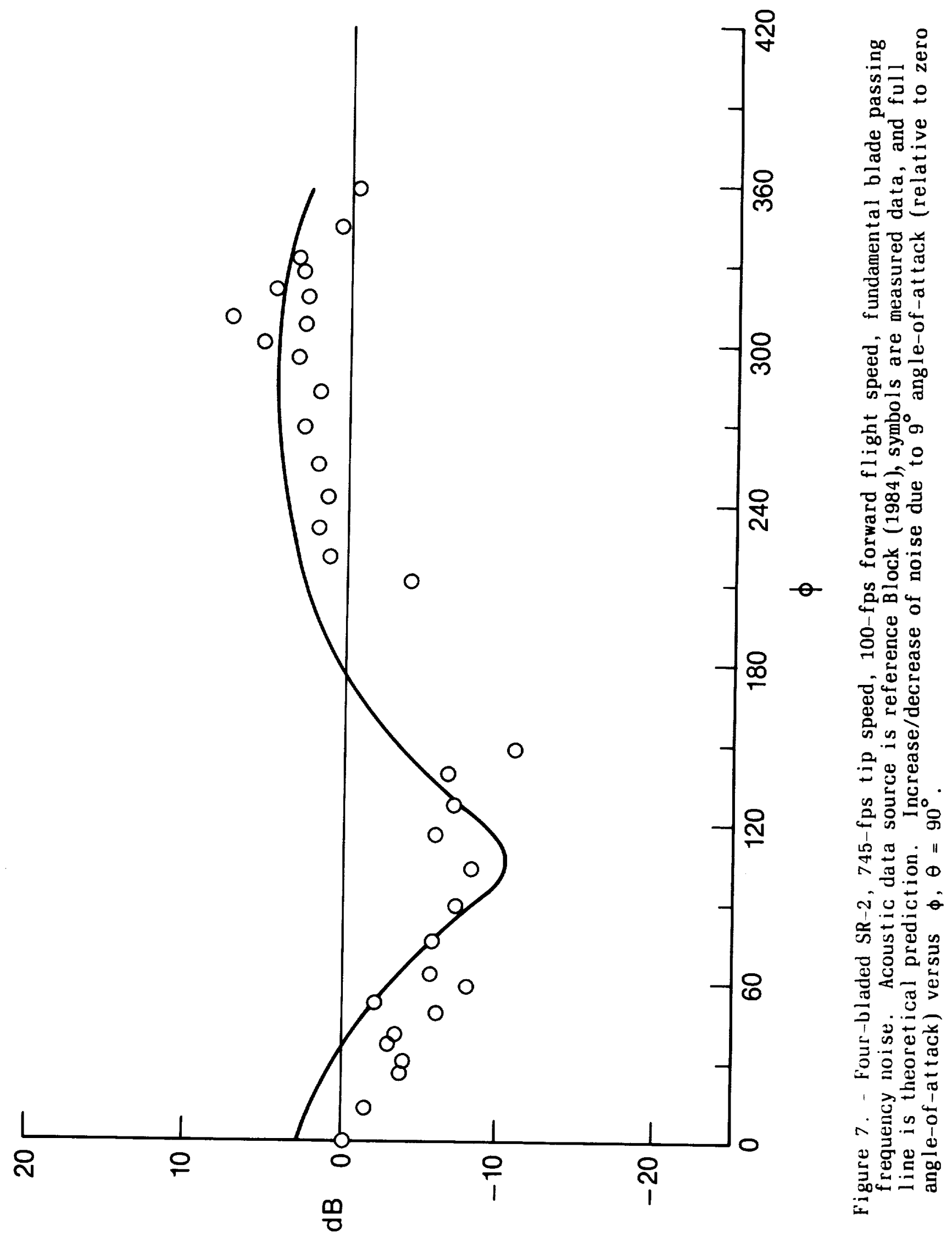




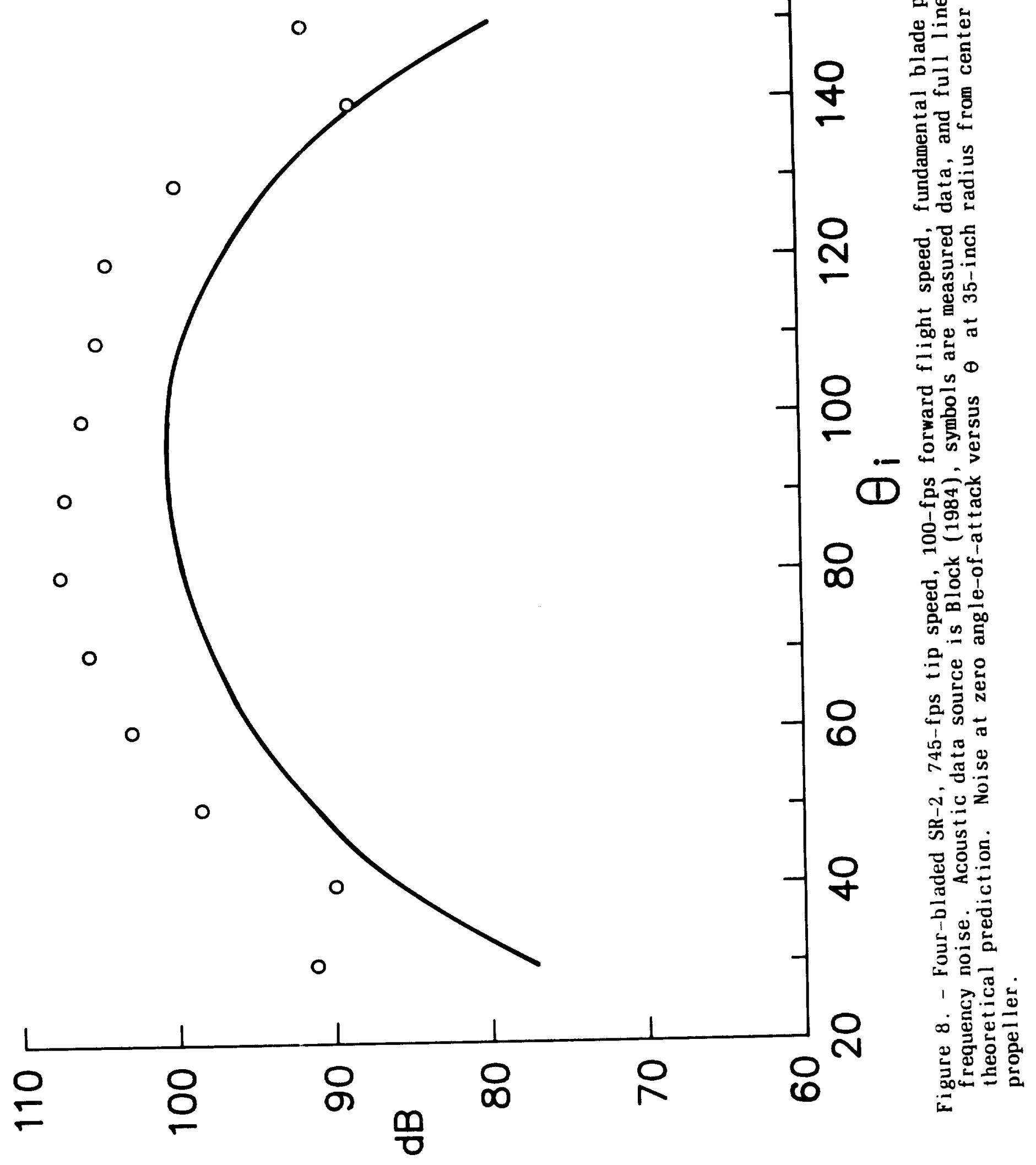




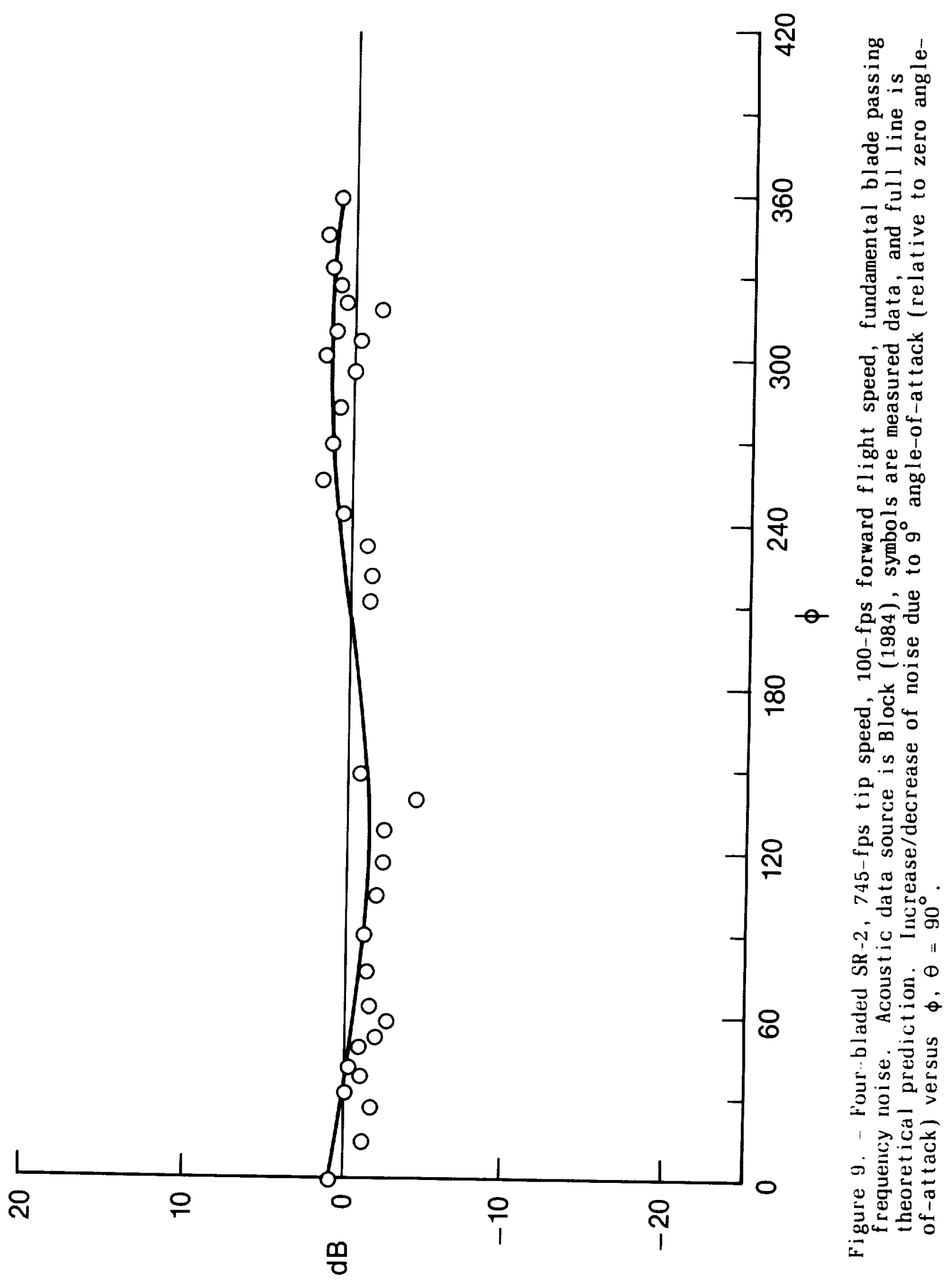




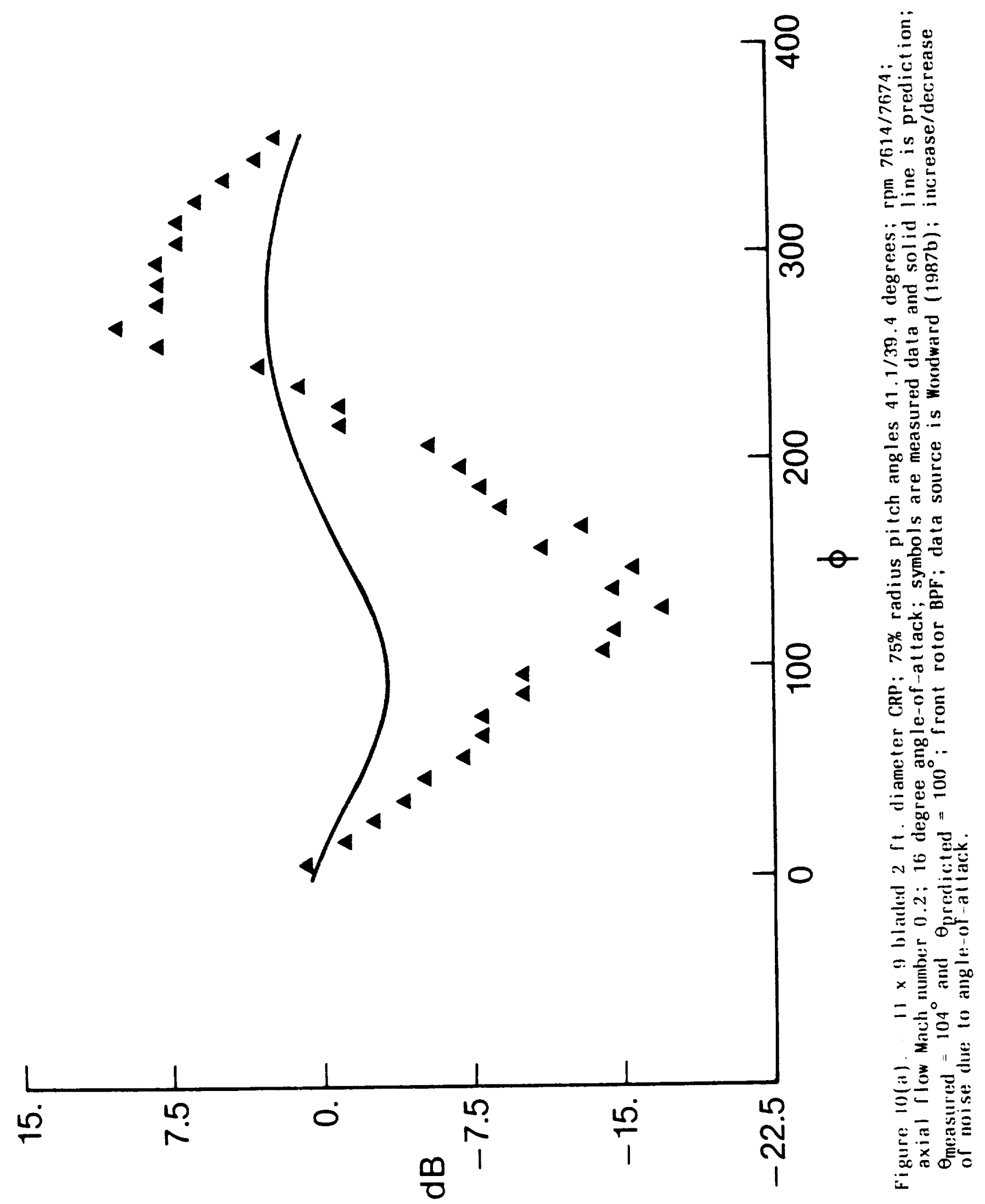




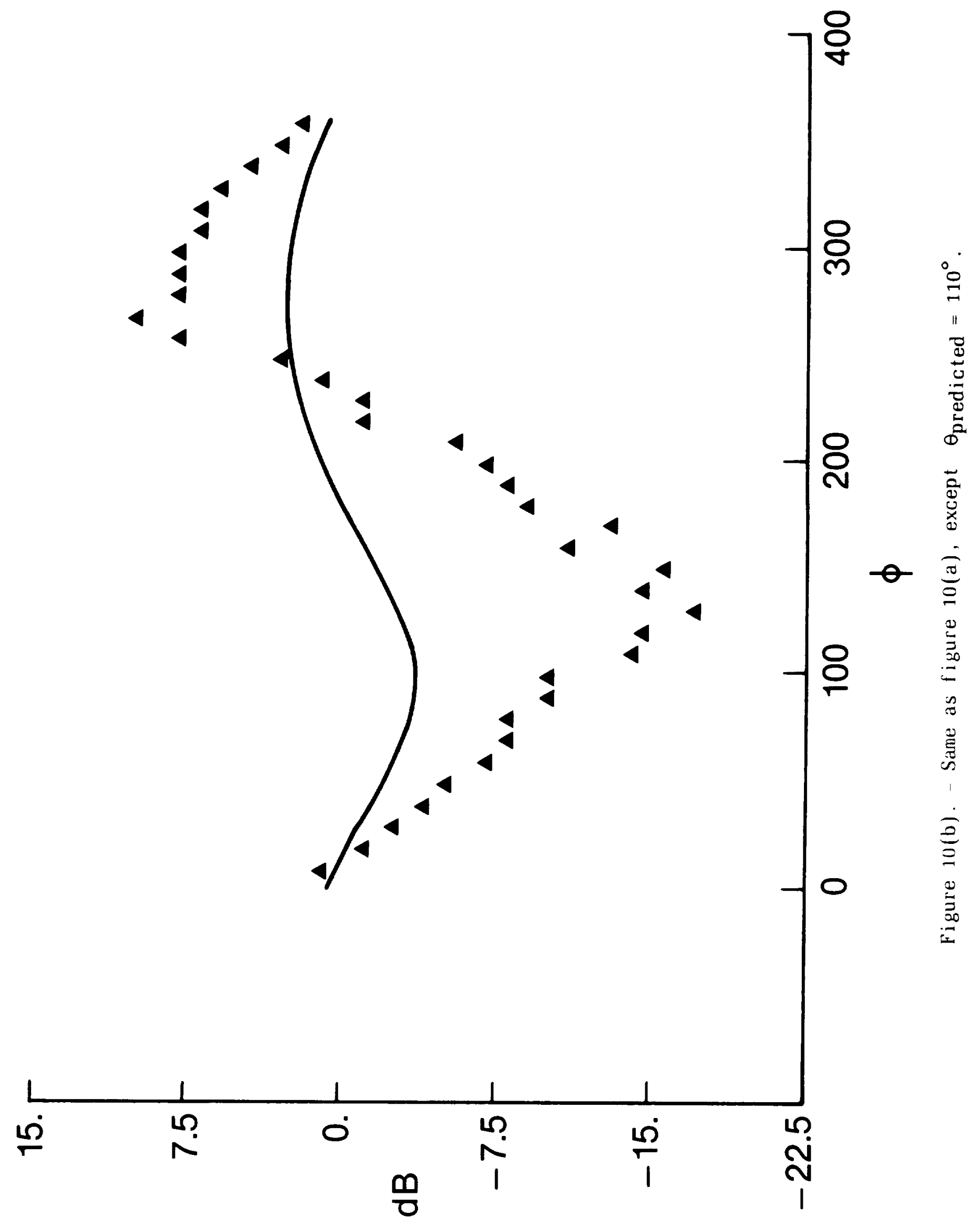



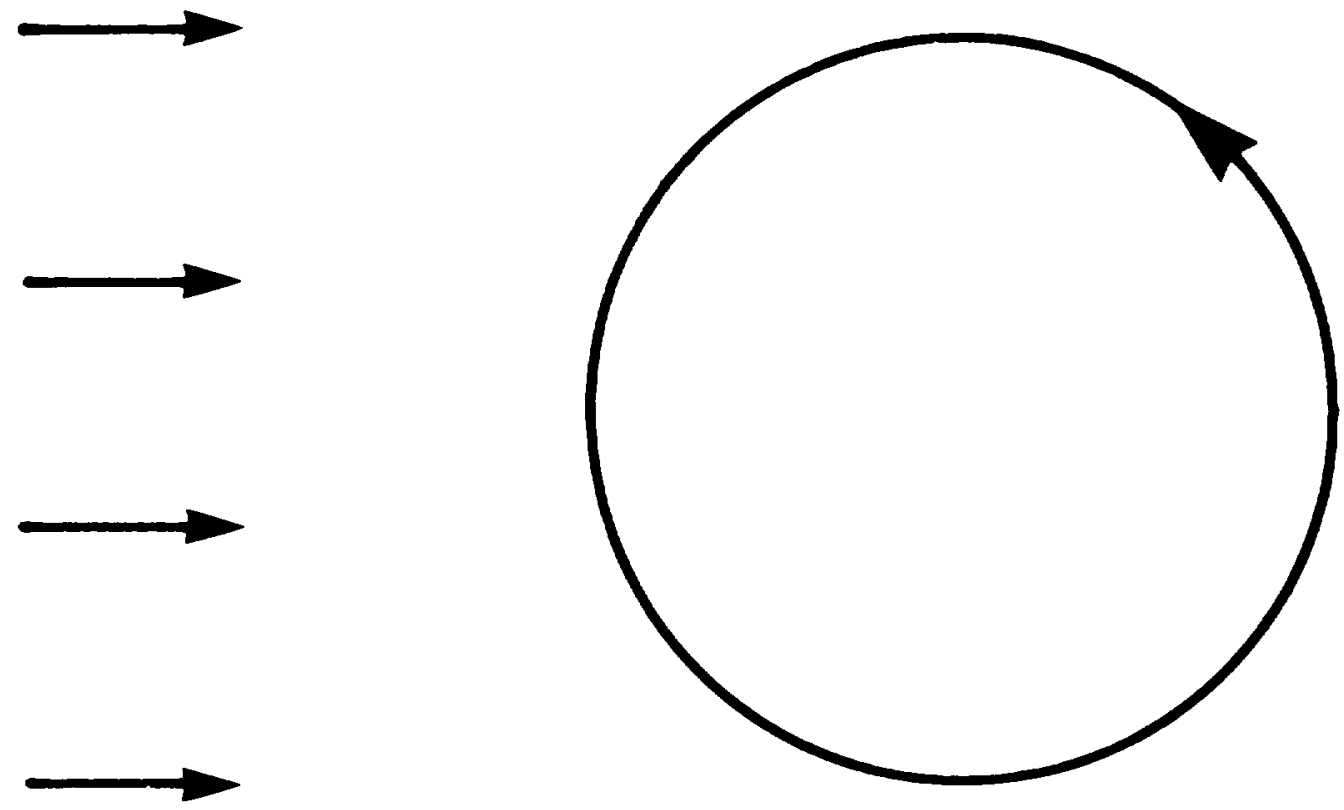

Figure 11.- Modulation of radiation efficiency of a rotating steady source due to cross flow. 


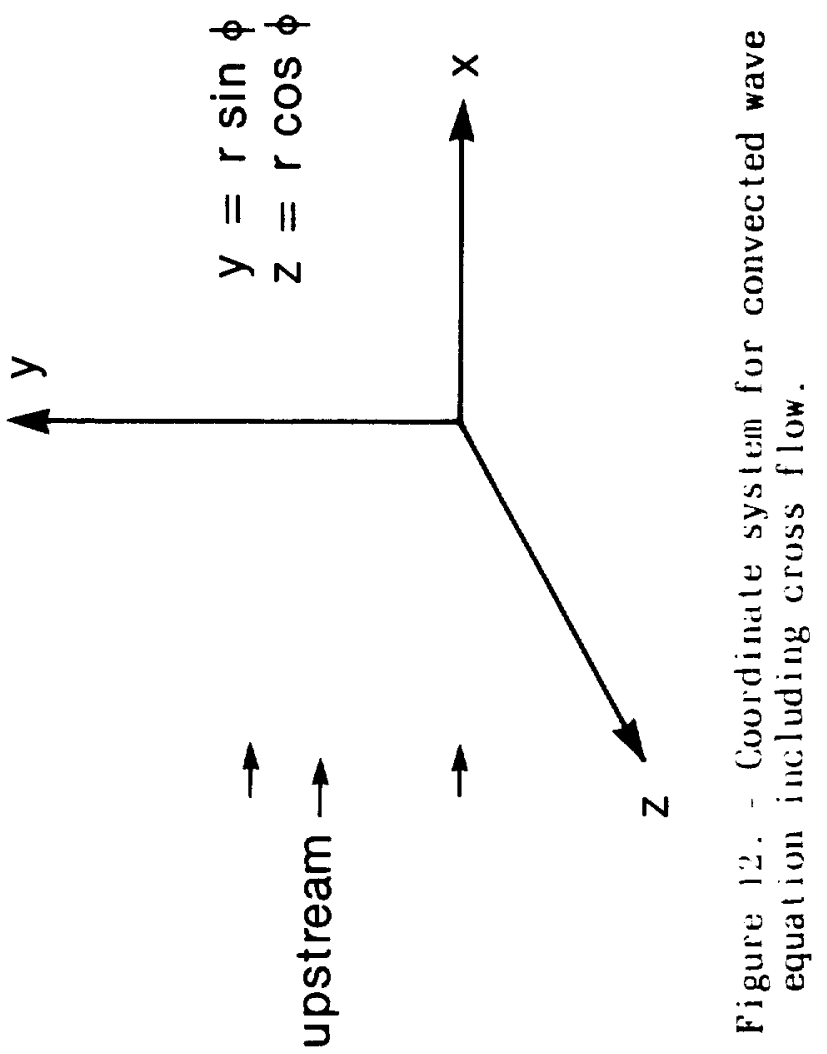




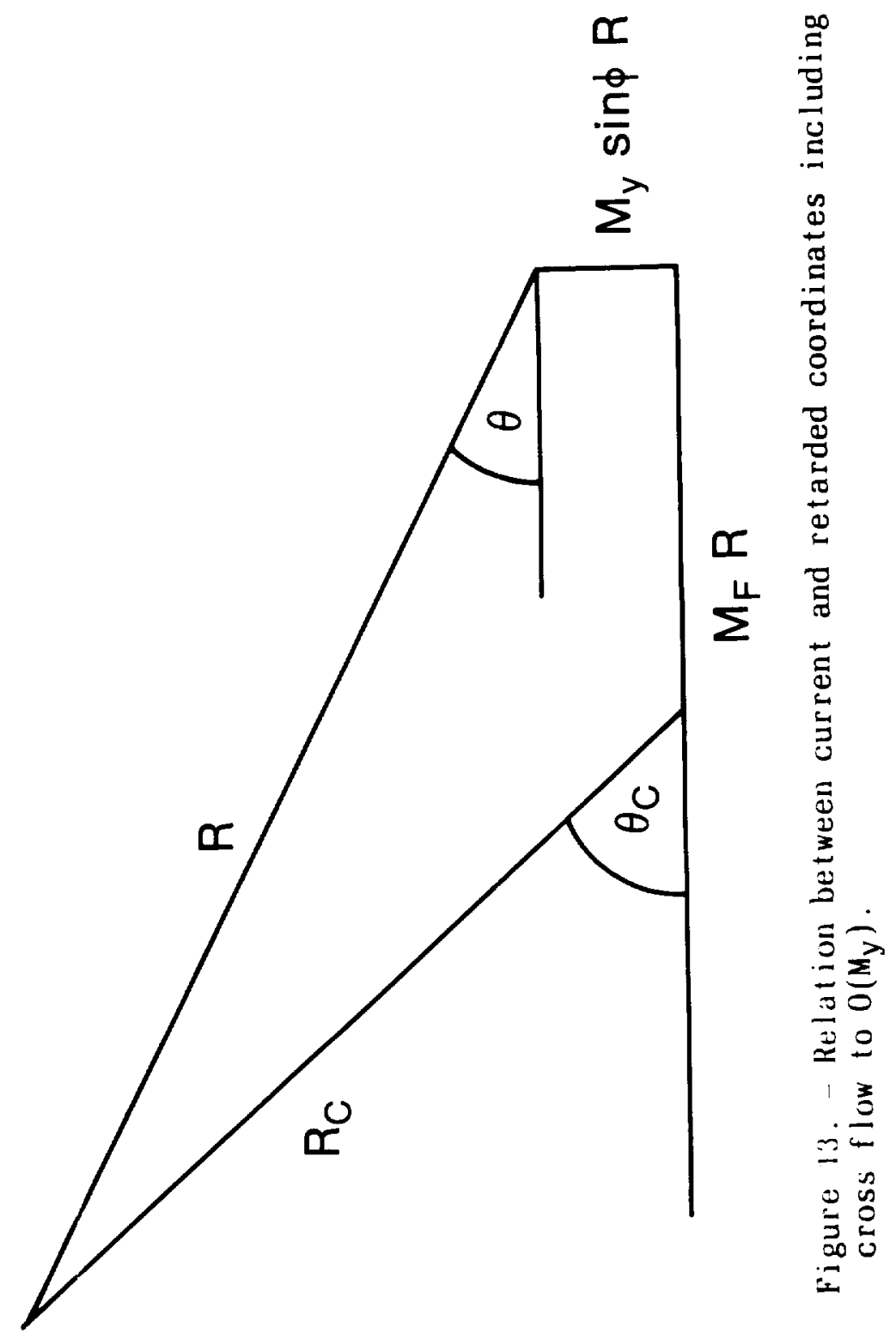




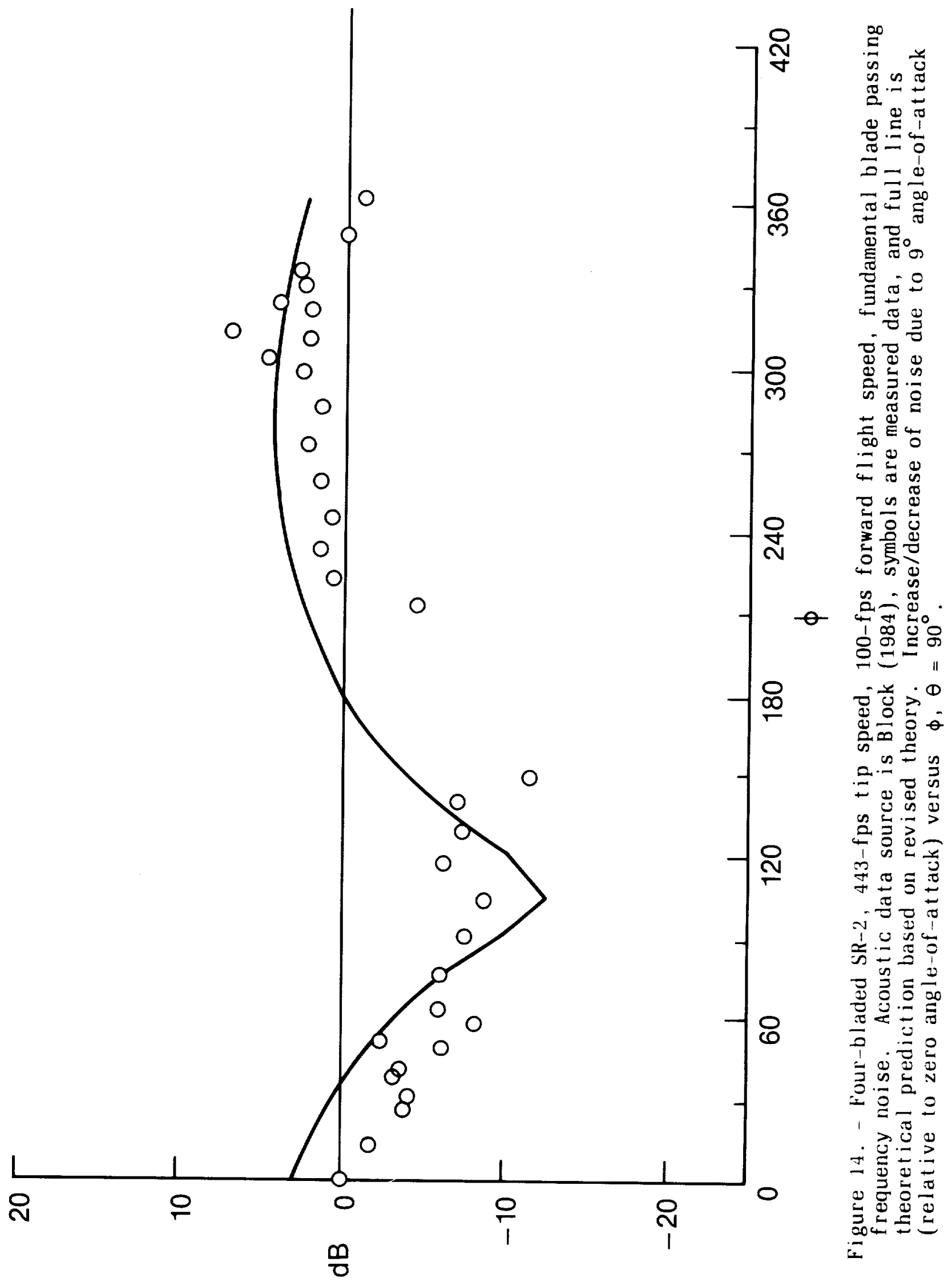




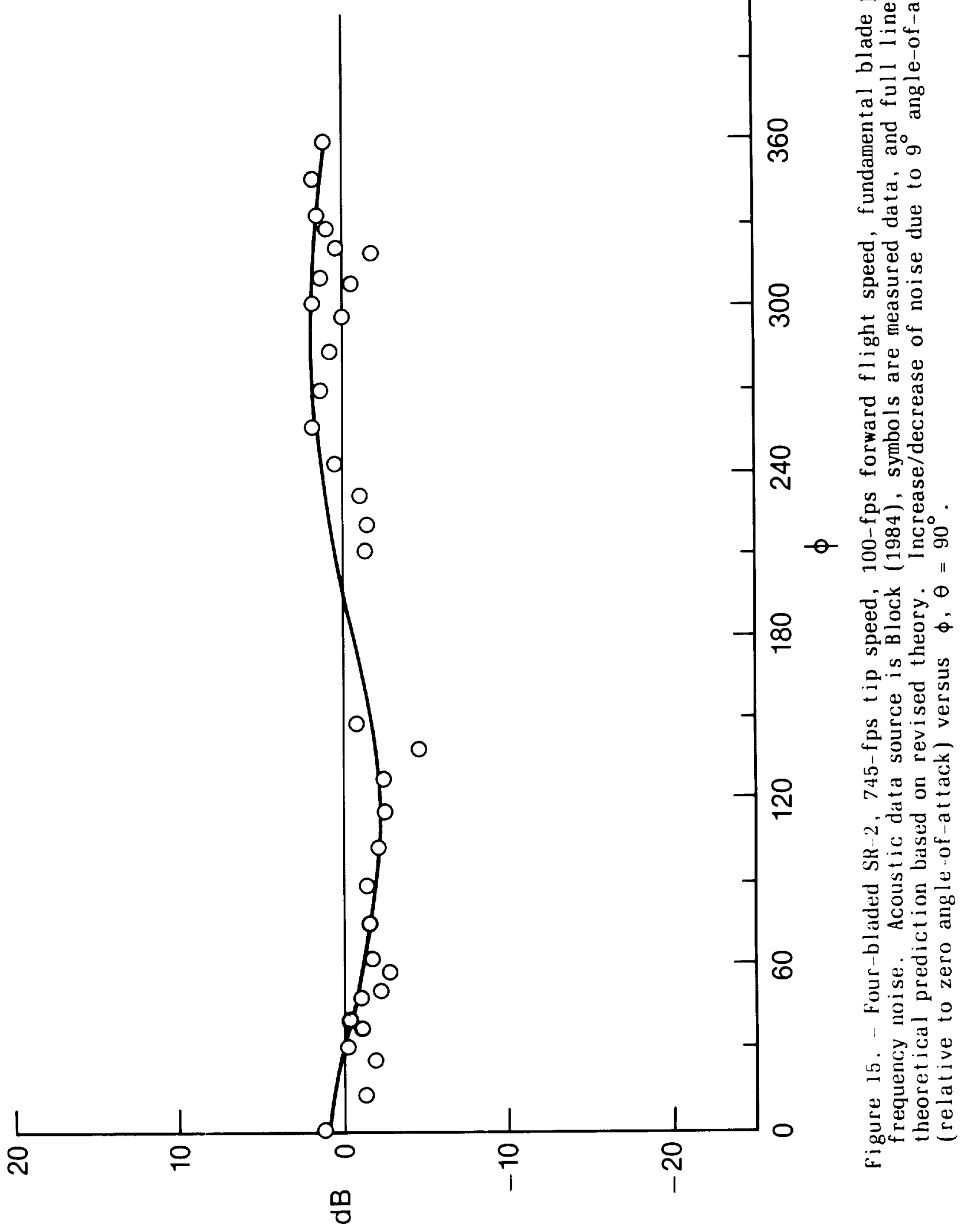




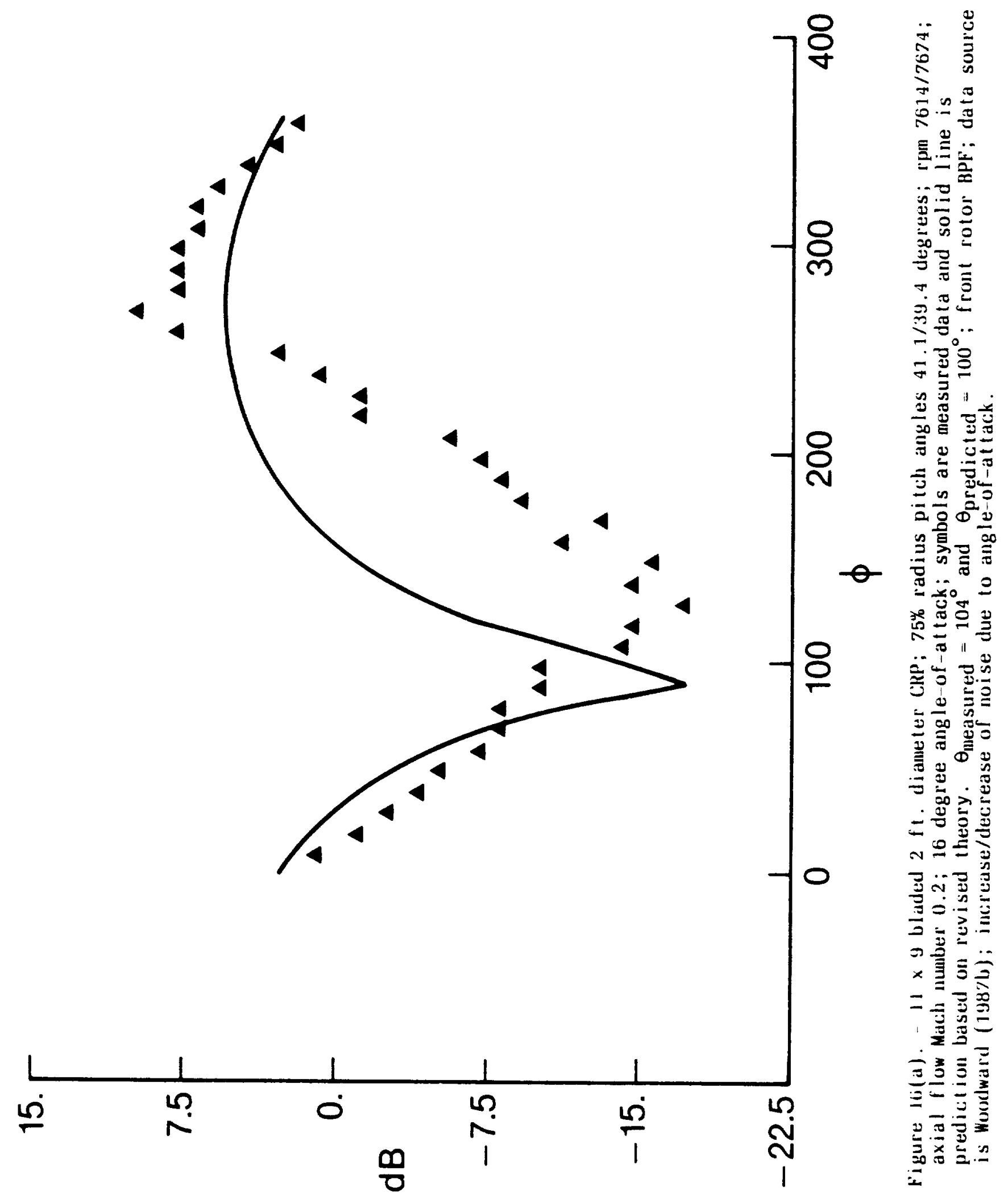




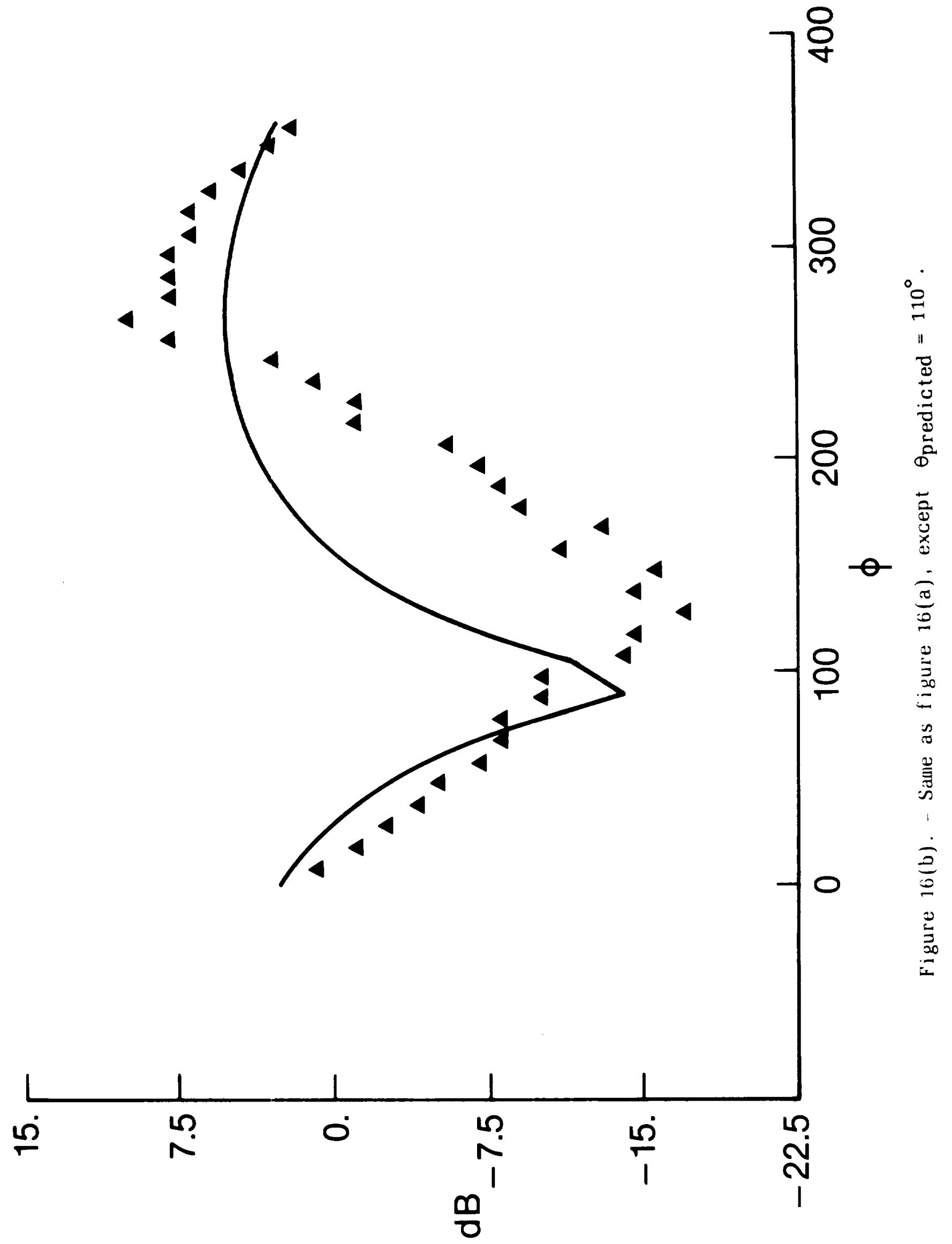




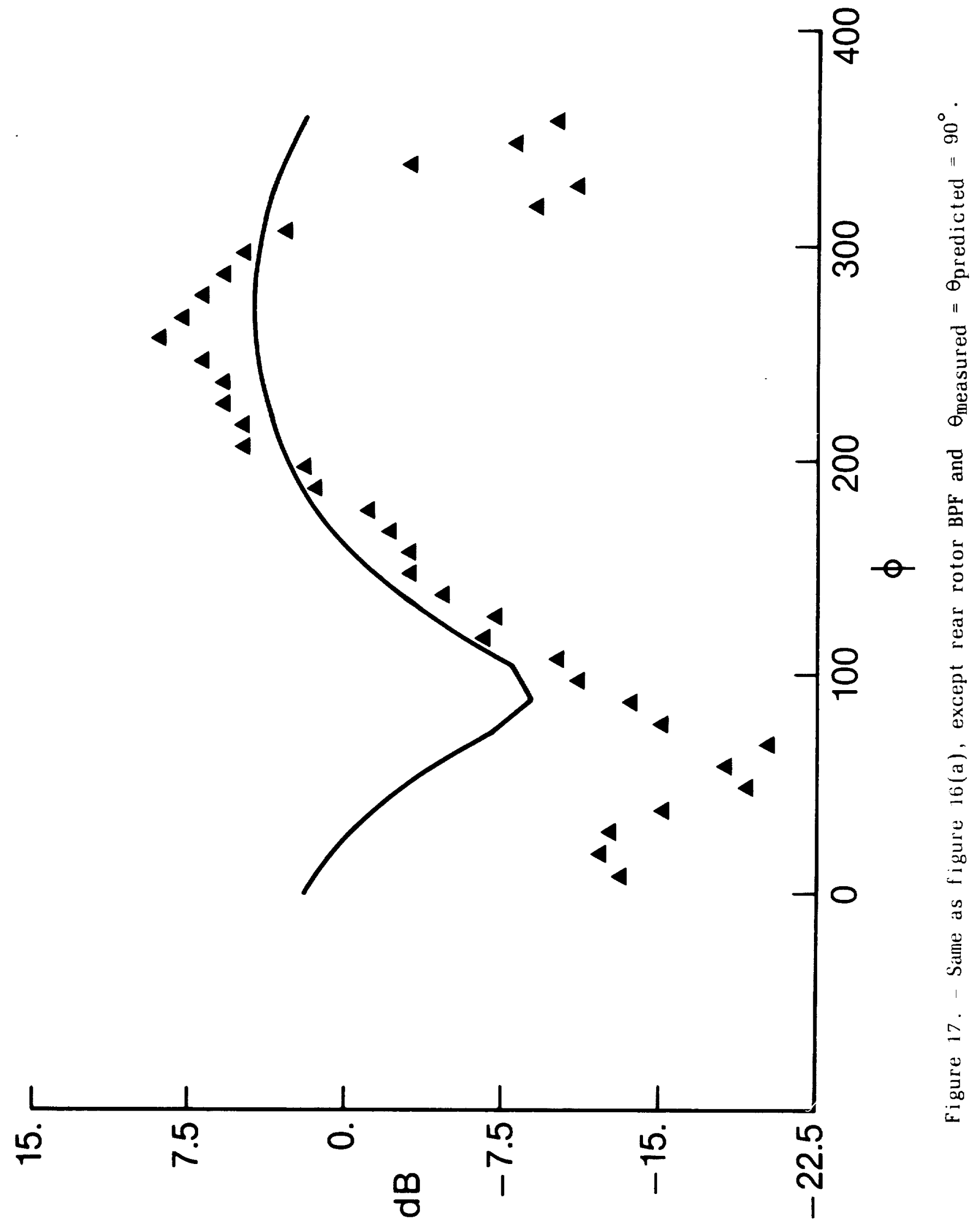




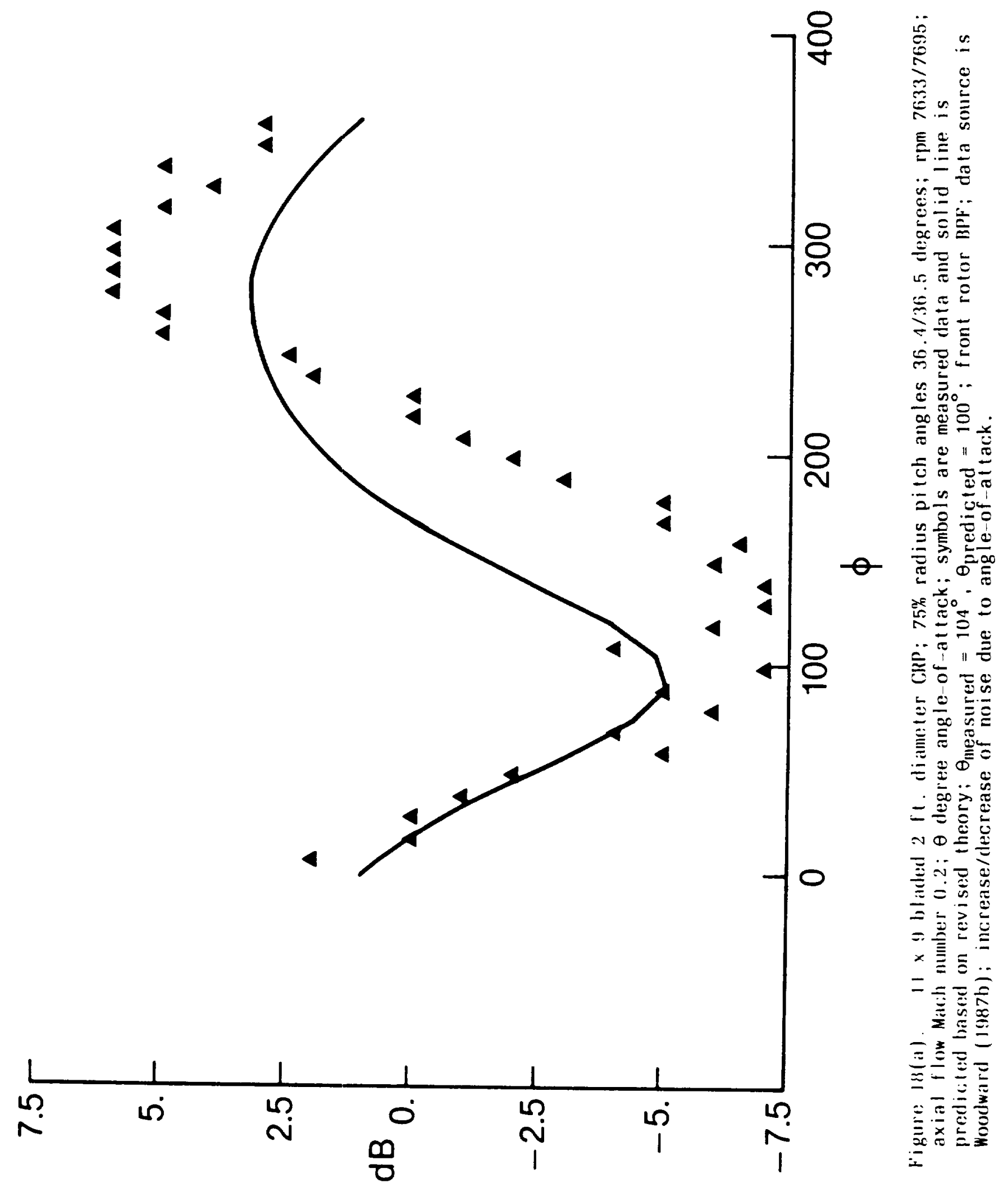




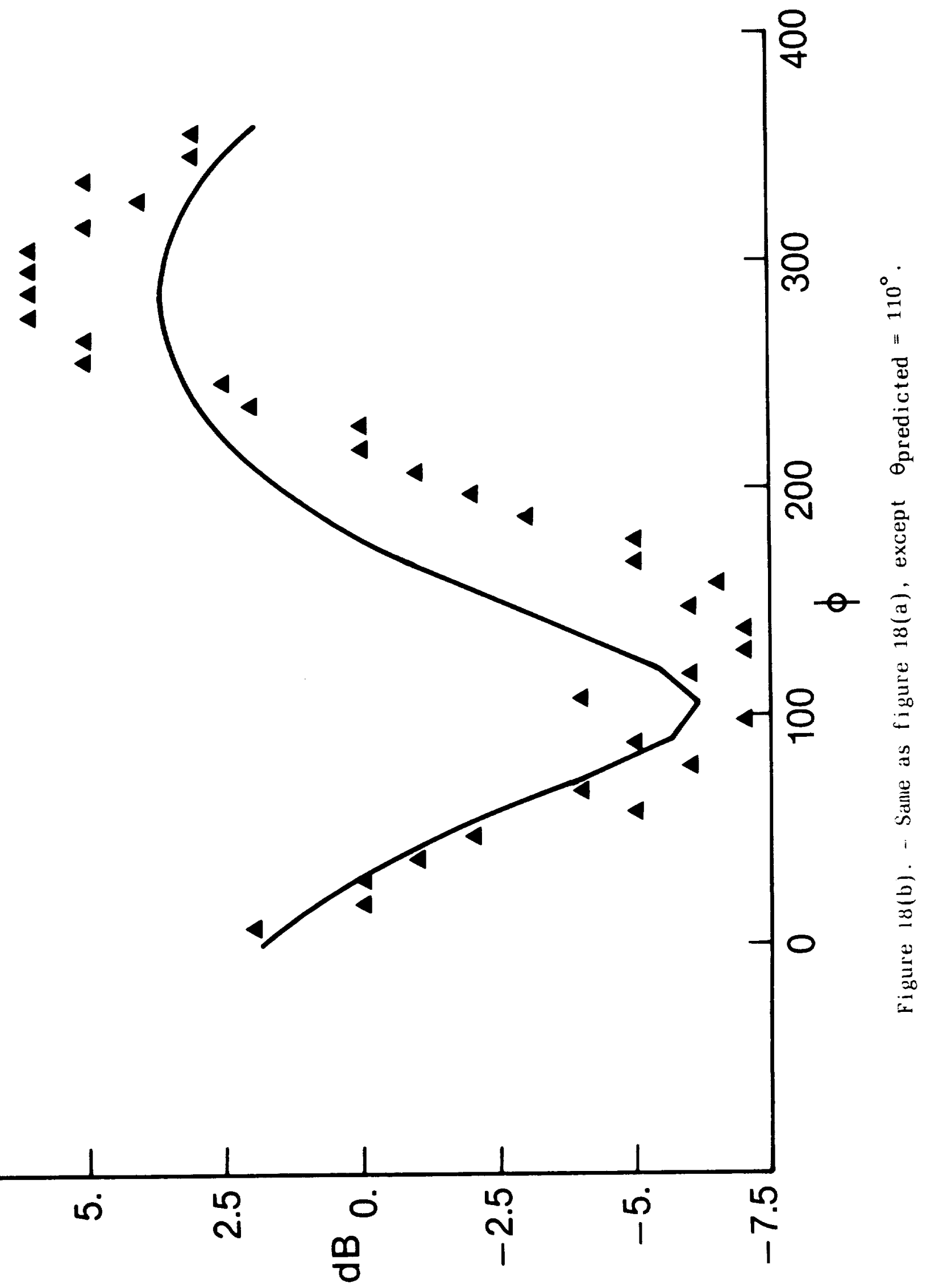




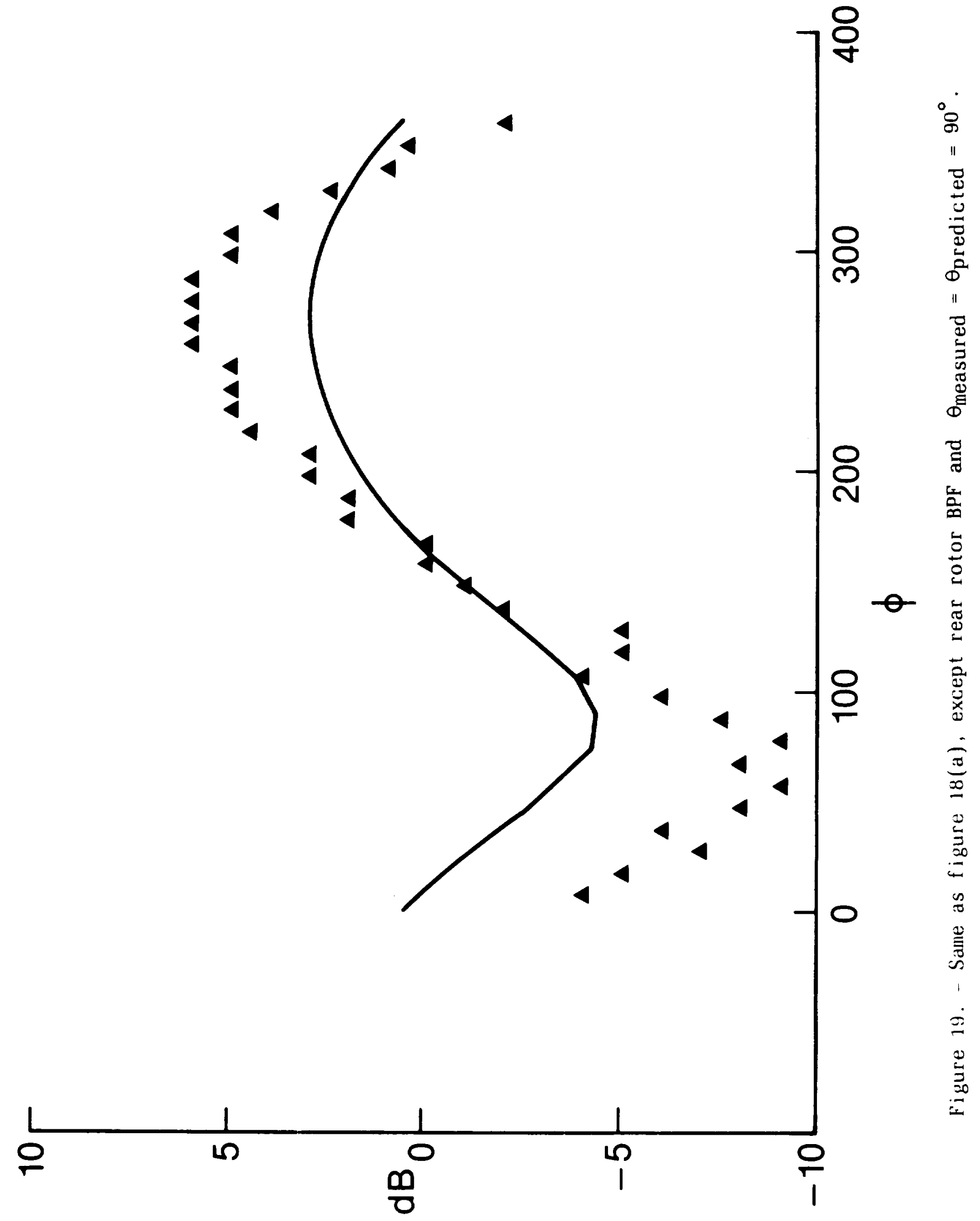




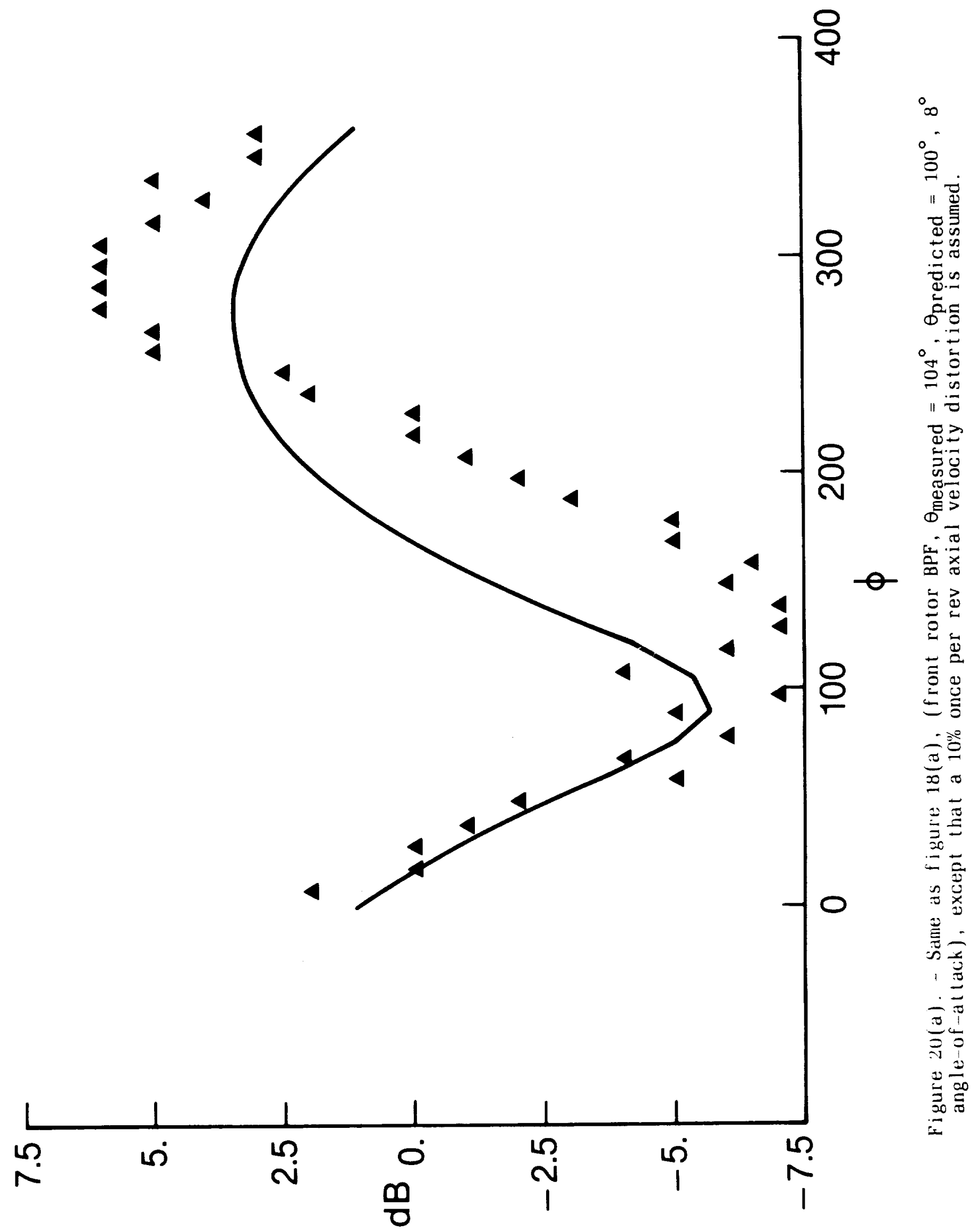




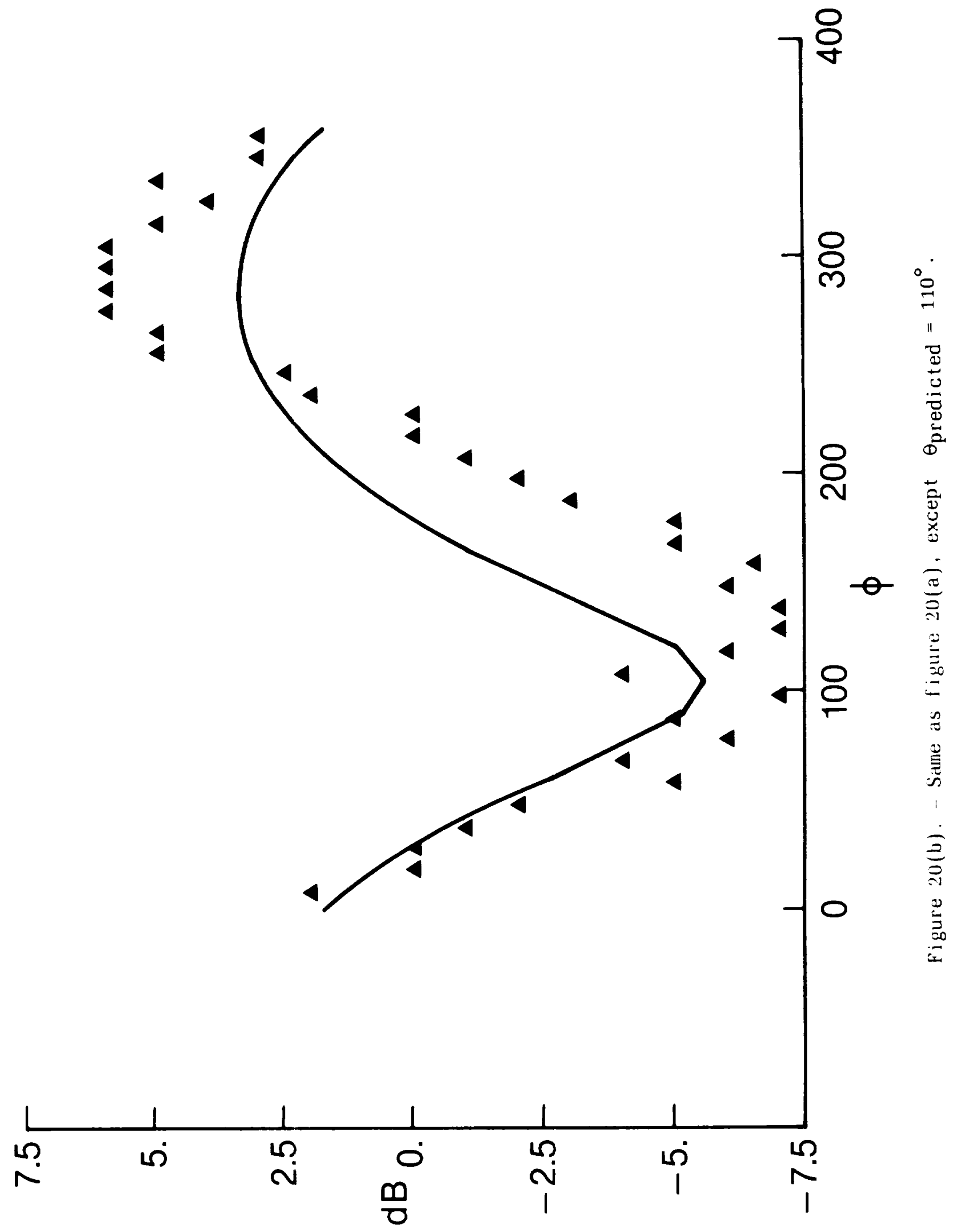




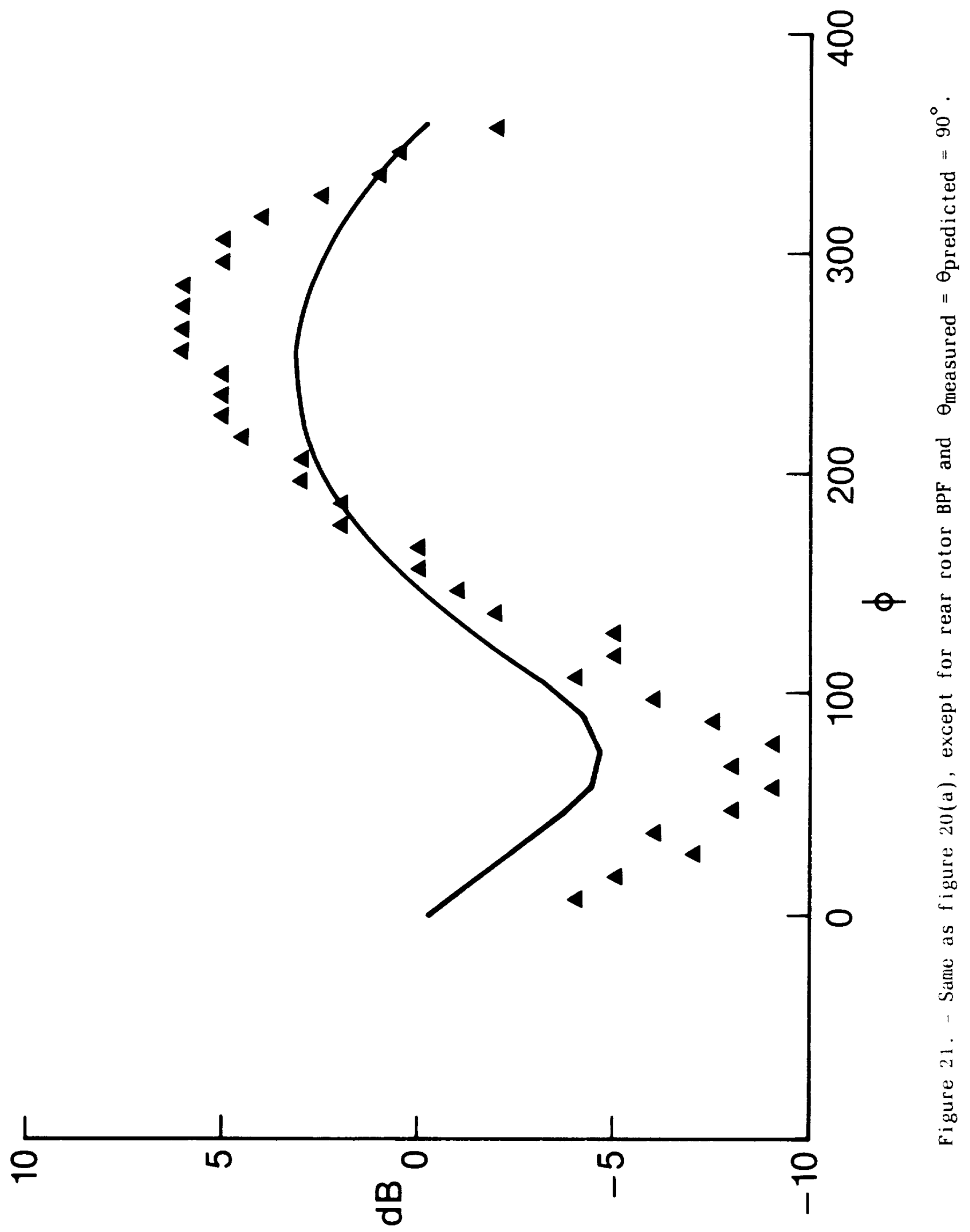




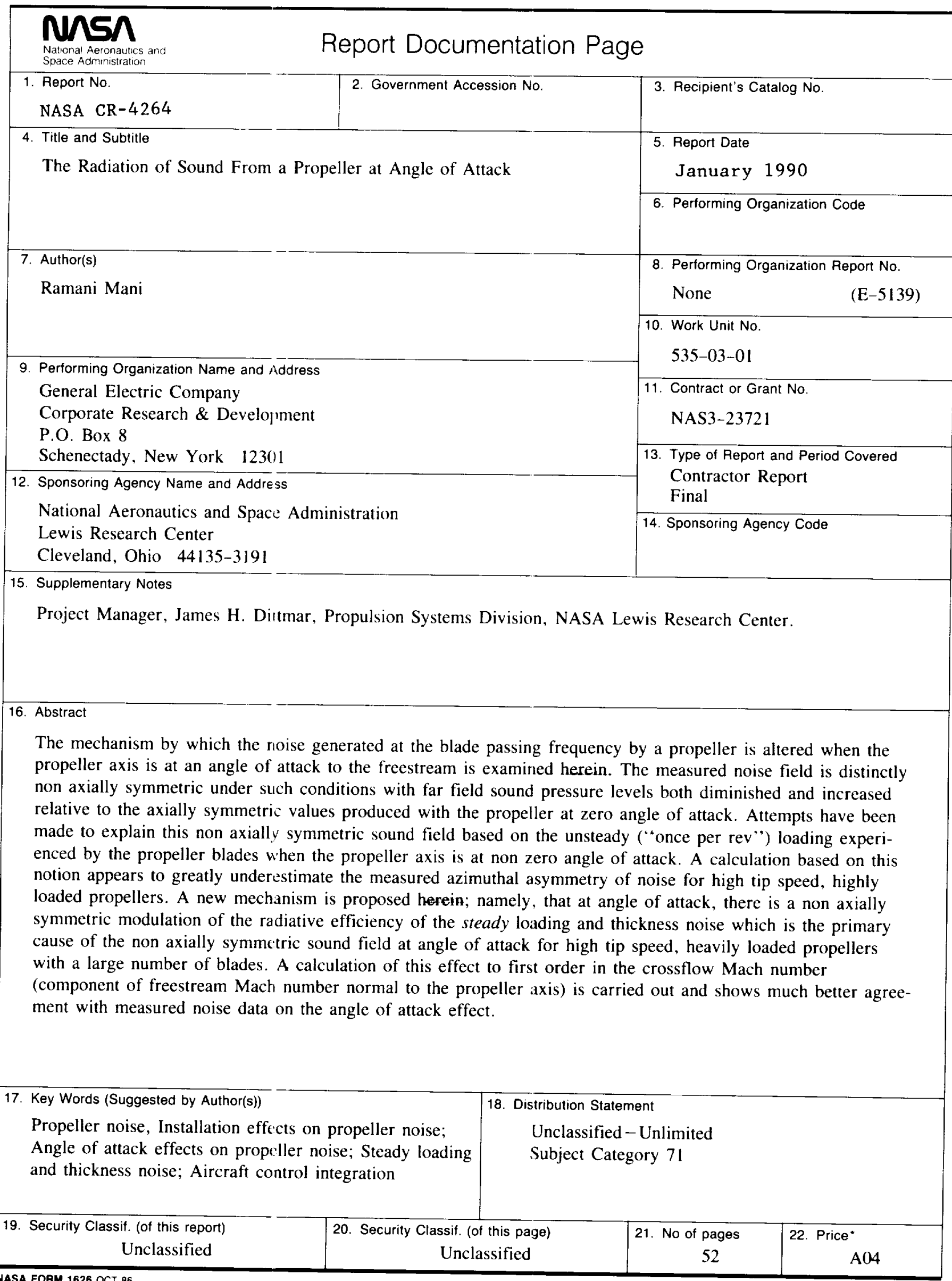


$-2-1-3$
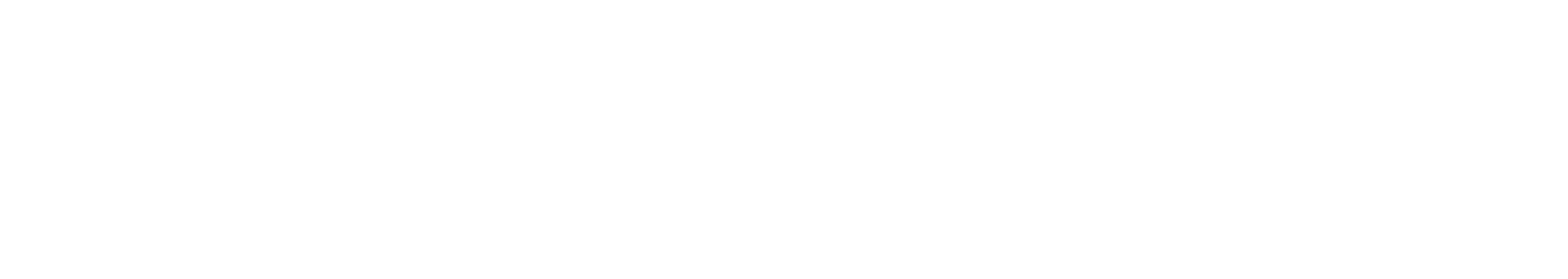

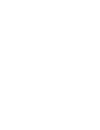
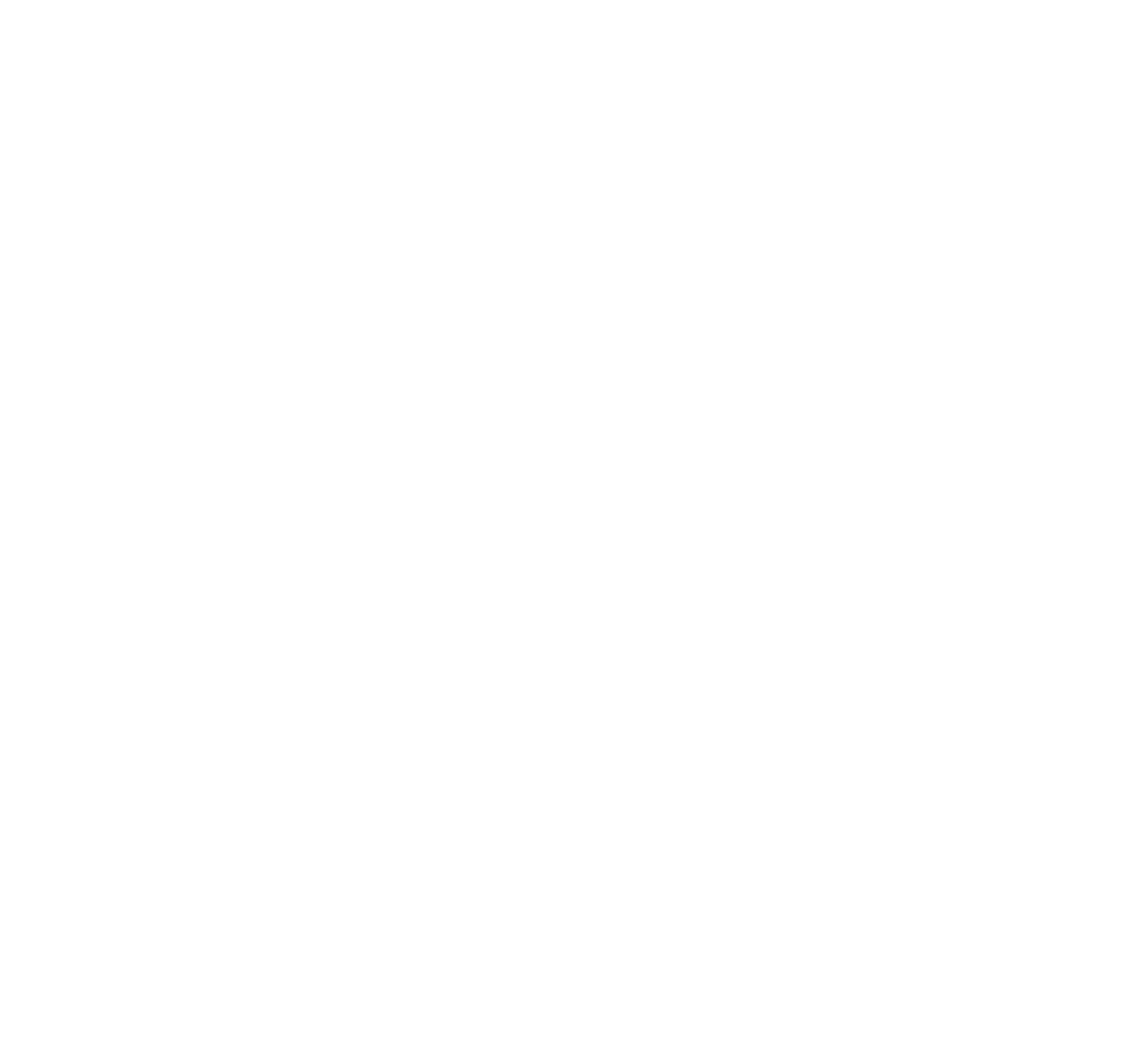